\title{
Equivalent Conditions for Jacobian Nonsingularity in Linear Symmetric Cone Programming*
}

\author{
Lingchen Kong ${ }^{1}$, Levent Tunçel ${ }^{2}$, and Naihua Xiu ${ }^{3}$
}

(October 07, 2008; Revised June 1, 2010)

\begin{abstract}
In this paper we consider the linear symmetric cone programming (SCP). At a KarushKuhn-Tucker (KKT) point of SCP, we present the important equivalent conditions for the nonsingularity of Clarke's generalized Jacobian of the KKT nonsmooth system, such as primal and dual constraint nondegeneracy, the strong regularity, and the nonsingularity of the B-subdifferential of the KKT system. This affirmatively answers an open question by Chan and Sun [SIAM J. Optim. 19 (2008), pp. 370-396].
\end{abstract}

Keywords: Linear symmetric cone programming, nonsingularity, constraint nondegeneracy, strong regularity, variational analysis.

AMS Subject Classification: 90C25, 90C22, 65K05, 65K10

Abbreviated Title: Jacobian Nonsingularity in SCP

\section{Introduction}

Consider the linear symmetric cone programming (SCP for short) as follows:

$$
\begin{array}{cc}
\min & \langle c, x\rangle \\
\text { s.t. } & \mathcal{A}(x)=b, \\
& x \in K,
\end{array}
$$

where $c \in \mathcal{V}, \mathcal{V}:=(\mathcal{J},\langle\cdot, \cdot\rangle, \circ)$ is a $n$-dimensional Euclidean Jordan algebra (see Section 2), $K$ is the symmetric cone in $\mathcal{V}, \mathcal{A}: \mathcal{V} \rightarrow \mathbb{R}^{m}$ is a linear operator, and $b \in \mathbb{R}^{m}$. The SCP provides a simple, natural, and unified framework for various existing optimization problems, which includes the linear programming (LP), the second-order cone programming (SOCP), the semidefinite programming (SDP) problems, and has wide applications in engineering, economics, management science, and other fields.

\footnotetext{
${ }^{*}$ The work was partly supported by a Discovery Grant from NSERC, and the National Natural Science Foundation of China (10831006) and the National Basic Research Program of China (2010CB732501).

${ }^{1}$ Lingchen Kong, Department of Applied Mathematics, Beijing Jiaotong University, Beijing 100044, P. R. China; Department of Combinatorics and Optimization, Faculty of Mathematics, University of Waterloo, Waterloo, Ontario N2L 3G1, Canada (e-mail: konglchen@126.com)

${ }^{2}$ Levent Tunçel, Department of Combinatorics and Optimization, Faculty of Mathematics, University of Waterloo, Waterloo, Ontario N2L 3G1, Canada (e-mail: ltuncel@math.uwaterloo.ca)

${ }^{3}$ Naihua Xiu, Department of Applied Mathematics, Beijing Jiaotong University, Beijing 100044, P. R. China (e-mail: nhxiu@center.njtu.edu.cn)
} 
Let $\mathcal{A}^{*}: \mathbb{R}^{m} \rightarrow \mathcal{V}$ be the adjoint operator of $\mathcal{A}$. Then, the dual of the SCP problem (1) is given by

$$
\begin{array}{cc}
\max & b^{T} y \\
\text { s.t. } & \mathcal{A}^{*}(y)+s=c, \\
& s \in K .
\end{array}
$$

Thus, the KKT conditions of the SCP problem (1) and its dual (2) become the following:

$$
\left\{\begin{array}{l}
\mathcal{A}(x)=b \\
\mathcal{A}^{*}(y)+s=c \\
x \in K, s \in K,\langle x, s\rangle=0 .
\end{array}\right.
$$

We call $(\bar{x}, \bar{y}, \bar{s}) \in \mathcal{V} \times \mathbb{R}^{m} \times \mathcal{V}$ a $K K T$ point if it satisfies the KKT conditions (3). Note that $(\bar{x}, \bar{y}, \bar{s})$ is a KKT point if and only if it is a solution to the KKT (nonsmooth) system:

$$
H(x, y, s):=\left(\begin{array}{c}
c-\mathcal{A}^{*}(y)-s \\
\mathcal{A}(x)-b \\
x-(x-s)_{+}
\end{array}\right)=0, \quad(x, y, s) \in \mathcal{V} \times \mathbb{R}^{m} \times \mathcal{V}
$$

where $(\cdot)_{+}$is the metric projection onto $K$. (The above equivalence can be traced back to Eaves [1] in the setting of complementarity problems; and in the special case of SCP, see also Gowda et. al [2]). It is well-known that nonsingularity of Clarke's generalized Jacobian of the KKT system, which is also called BD-regularity introduced by Pang and Qi [3], is not only one of the fundamental characterizations for sensitivity and stability analysis of optimization problems but also plays an important role in the design of algorithms, see [4] and the references therein. For sensitivity and stability analysis of optimization problems, see [5] and the references therein. This paper deals with the nonsingularity of Clarke's generalized Jacobian of the KKT system (4) in the setting of SCP. In particular, we consider its connection to another important concept, nondegeneracy (primal and dual nondegeneracy and weak nondegeneracy), which has been extensively studied in the settings of SDP and SOCP, see, e.g., [6, 7, 8, 9, 10, 11, 12, 13] and even developed in the general setting of optimization problems over arbitrary convex cones $[14,15,16]$. For instance, Pataki [14] introduced the notions of primal and dual nondegeneracy of convex optimization problems in conic form and showed that at a primal-dual optimal solution, primal (dual) nondegeneracy implies the uniqueness of the dual (primal) optimal solution (this is also independently established by Shapiro and Fan [17]) and the reverse implication holds only under strict complementarity; Pataki and Tunçel [15] proved that the primal and dual nondegeneracy, and strict complementarity are all generic properties. (The results mentioned above generalize the corresponding results of Alizadeh-Haeberly-Overton [6] from the setting of SDP.) Moreover, Yildirim [13] introduced the notion of weak primal and dual nondegeneracy in the SDP context, and then developed it in the general conic form and showed that the weak primal (dual) nondegeneracy is necessary and sufficient for the existence of a unique dual (primal) optimal solution [16]. Recently, in the SDP context, Chan and Sun [8] firstly showed that at a KKT point, nonsingularity of Clarke's generalized Jacobian of the KKT system is equivalent to the nonsingularity of its B-subdifferential and some other important conditions such as the primal and dual constraint nondegeneracy, and the strong regularity. In the same paper, Chan and Sun asked whether the corresponding results for SDP can be extended to SCP.

In this paper we answer the above question in the affirmative. To do so, we develop a new technique which serves as a fundamental tool for our analysis in this paper. More precisely, we 
provide a decomposition result of Euclidean Jordan algebras, and establish a triangular representation of the Jacobian of Löwner operators. Then, we present the triangular representation of Clarke's generalized Jacobian of the projection operator onto symmetric cone $K$ and give an explicit formula for the tangent cone of $K$. This helps us prove the equivalence at a KKT point of the nonsingularity of Clarke's generalized Jacobian of the KKT system (4), the nonsingularity of its B-subdifferential, the primal and dual constraint nondegeneracy, and the strong regularity.

As we were sharing the results and the earlier drafts of this paper with some colleagues ${ }^{\S}$, we learned that Wang [18] independently obtained, around the same time, essentially the same main result. Her approach is based on the connection between the Lyapunov transformation (operator) and its matrix representation with respect to the orthonormal basis of $\mathcal{V}$. The technique used in our proof below is established on the exact expression for Clarke's generalized Jacobian of $\Pi_{K}$ and a decomposition result of Euclidean Jordan algebras (see Section 2) based on a generalized weighted partial trace operator which may have other applications. We also expose the intimate connections among the main result, Robinson's notion of constraint nondegeneracy and the geometric notions of nondegeneracy (see Bonnans and Shapiro [5], Pataki [14]) in general convex optimization problems in conic form (see Section 3).

This paper is organized as follows. In Section 2, we briefly describe some fundamental concepts and the decomposition results on Euclidean Jordan algebras. We give the triangular representations of the Jacobian of Löwner operators and Clarke's generalized Jacobian of projection operator onto symmetric cone. Then, we present the tangent cone and some of its properties used in our analysis. In Section 3, we show our main equivalence result.

\section{Preliminaries}

\subsection{Euclidean Jordan algebras}

We review some necessary results on Euclidean Jordan algebras details of which can be found in Koecher's lecture notes [19] and the monograph by Faraut and Korányi [20].

Let $\mathcal{J}$ be a $n$-dimensional vector space over $\mathbb{R}$ and $(x, s) \mapsto x \circ s: \mathcal{J} \times \mathcal{J} \rightarrow \mathcal{J}$ be a bilinear mapping. We call $(\mathcal{J}, \circ)$ a Jordan algebra if the bilinear mapping satisfies the following conditions:

(i) $x \circ s=s \circ x$ for all $x, s \in \mathcal{J}$,

(ii) $x \circ\left(x^{2} \circ s\right)=x^{2} \circ(x \circ s)$ for all $x, s \in \mathcal{J}$,

where $x^{2}:=x \circ x$ and $x \circ s$ is the Jordan product of $x$ and $s$. In general, there may exist $x, s, z \in \mathcal{J}$ such that $(x \circ s) \circ z \neq x \circ(s \circ z)$. We call an element $e$ the identity element if $z \circ e=e \circ z=z$ for all $z \in \mathcal{J}$. A Jordan algebra $(\mathcal{J}, \circ)$ with an identity element $e$ is called a Euclidean Jordan algebra, denoted by $\mathcal{V}:=(\mathcal{J},\langle\cdot, \cdot\rangle, \circ)$, if there is an inner product, $\langle\cdot, \cdot\rangle$, such that

$$
\langle x \circ s, z\rangle=\langle x, s \circ z\rangle \text { for all } x, s, z \in \mathcal{J} \text {. }
$$

Given a Euclidean Jordan algebra $\mathcal{V}$, define the set of squares as $K:=\left\{z^{2}: z \in \mathcal{V}\right\}$. It is known by Theorem III 2.1 in [20] that $K$ is the symmetric cone, i.e., $K$ is a closed, convex, homogeneous and self-dual cone.

For $z \in \mathcal{V}$, the degree of $z$ denoted by $\operatorname{deg}(z)$ is the smallest positive integer $k$ such that the set $\left\{e, z, z^{2}, \cdots, z^{k}\right\}$ is linearly dependent. The $\operatorname{rank}$ of $\mathcal{V}$ is defined as $\max \{\operatorname{deg}(z): z \in \mathcal{V}\}$. In

\footnotetext{
${ }^{\S}$ We thank Defeng Sun for pointing out the reference [18] and Liwei Zhang for sending us Wang's PhD thesis.
} 
this paper, $r$ will denote the rank of the underlying Euclidean Jordan algebra. An element $q \in \mathcal{V}$ is an idempotent if $q^{2}=q \neq 0$, which is also called primitive if it cannot be written as a sum of two idempotents. A complete system of orthogonal idempotents is a finite set $\left\{q_{1}, q_{2}, \cdots, q_{k}\right\}$ of idempotents where $q_{i} \circ q_{j}=0$ for all $i \neq j$, and $q_{1}+q_{2}+\cdots+q_{k}=e$. A Jordan frame is a complete system of orthogonal primitive idempotents in $\mathcal{V}$. Note that the number of elements in every Jordan frame is $r$.

We state below the spectral decomposition theorem for the elements in a Euclidean Jordan algebra.

Theorem 2.1 (Spectral Decomposition Type II (Theorem III.1.2, [20])) Let $\mathcal{V}$ be a Euclidean Jordan algebra with rank $r$. Then for $z \in \mathcal{V}$ there exist a Jordan frame $\left\{q_{1}, q_{2}, \cdots, q_{r}\right\}$ and real numbers $\lambda_{1}(z) \geq \lambda_{2}(z) \geq \cdots \geq \lambda_{r}(z)$ such that

$$
z=\lambda_{1}(z) q_{1}+\lambda_{2}(z) q_{2}+\cdots+\lambda_{r}(z) q_{r}
$$

The numbers $\lambda_{i}(z)(i \in\{1,2, \cdots, r\})$ are the eigenvalues of $z$. We call (5) the spectral decomposition (or the spectral expansion) of $z$.

Observe that the Jordan frame $\left\{q_{1}, q_{2}, \cdots, q_{r}\right\}$ in (5) depends on $z$. We do not write this dependence explicitly for the simplicity of notation (the same for $\left\{e_{1}, e_{2}, \cdots, e_{\bar{r}}\right\}$ below). Let $\mathcal{C}(z)$ be the set consisting of all Jordan frames in the spectral decomposition of $z$. Let the spectrum $\sigma(z)$ be the set of all eigenvalues of $z$. Then $\sigma(z)=\left\{\mu_{1}(z), \mu_{2}(z), \cdots, \mu_{\bar{r}}(z)\right\}$ and for each $\mu_{i}(z) \in \sigma(z)$, denoting $N_{i}(z):=\left\{j: \lambda_{j}(z)=\mu_{i}(z)\right\}$ we obtain that $e_{i}=\sum_{j \in N_{i}(z)} q_{j}$ and $e_{i}$ is idempotent but may not be primitive. By Theorem III.1.1 in [20], $\left\{e_{1}, e_{2}, \cdots, e_{\bar{r}}\right\}$ is a unique complete system of orthogonal idempotents such that

$$
z=\mu_{1}(z) e_{1}+\cdots+\mu_{k}(z) e_{\bar{r}}
$$

Let $g: \mathbb{R} \rightarrow \mathbb{R}$ be a real-valued function. Define the vector-valued function $G: \mathcal{V} \rightarrow \mathcal{V}$ as

$$
G(z):=\sum_{i=1}^{r} g\left(\lambda_{i}(z)\right) q_{i}=g\left(\lambda_{1}(z)\right) q_{1}+g\left(\lambda_{2}(z)\right) q_{2}+\cdots+g\left(\lambda_{r}(z)\right) q_{r},
$$

which is a Löwner operator. In particular, letting $t_{+}:=\max \{0, t\}, t_{-}:=\min \{0, t\}(t \in \mathbb{R})$, we respectively define

$$
\Pi_{K}(z):=z_{+}:=\sum_{i=1}^{r}\left(\lambda_{i}(z)\right)_{+} q_{i}, \quad z_{-}:=\sum_{i=1}^{r}\left(\lambda_{i}(z)\right)_{-} q_{i} .
$$

In words, $z_{+}$is the metric projection of $z$ onto $K$, and $z_{-}$is the metric projection of $z$ onto $-K$, where the norm is defined by $\|z\|:=\sqrt{\langle z, z\rangle}$. Note that $z \in K(z \in \operatorname{int}(K))$ if and only if $\lambda_{i}(z) \geq 0\left(\lambda_{i}(z)>0\right) \forall i \in\{1,2, \cdots, r\}$, where $\operatorname{int}(K)$ denotes the interior of $K$. It is easy to verify that

$$
z_{+} \in K, \quad-z_{-} \in K, \quad z=z_{+}+z_{-} .
$$

Next, we recall the Peirce decomposition on the space $\mathcal{V}=(\mathcal{J},\langle\cdot, \cdot\rangle, \circ)$. Let $q \in \mathcal{V}$ be a nonzero idempotent. Then $\mathcal{V}$ is the orthogonal direct sum of $J(q, 0), J\left(q, \frac{1}{2}\right)$ and $J(q, 1)$, where

$$
J(q, \varepsilon):=\{x \in \mathcal{V}: q \circ x=\varepsilon x\}, \quad \varepsilon \in\left\{0, \frac{1}{2}, 1\right\} .
$$


This is called the Peirce decomposition of $\mathcal{V}$ with respect to the nonzero idempotent $q$. Fix a Jordan frame $\left\{q_{1}, q_{2}, \cdots, q_{r}\right\}$. Defining the following subspaces for $i, j \in\{1,2, \cdots, r\}$,

$$
J_{i i}:=\left\{x \in \mathcal{V}: x \circ q_{i}=x\right\} \text { and } J_{i j}:=\left\{x \in \mathcal{V}: x \circ q_{i}=\frac{1}{2} x=x \circ q_{j}\right\}, i \neq j,
$$

we have the Peirce decomposition theorem as follows.

Theorem 2.2 (Theorem IV.2.1, [20]) Let $\left\{q_{1}, q_{2}, \cdots, q_{r}\right\}$ be a given Jordan frame in a Euclidean Jordan algebra $\mathcal{V}$ of rank $r$. Then $\mathcal{V}$ is the orthogonal direct sum of spaces $J_{i j}(i \leq j)$. Furthermore,

(i) $J_{i j} \circ J_{i j} \subseteq J_{i i}+J_{j j}$;

(ii) $J_{i j} \circ J_{j k} \subseteq J_{i k}$, if $i \neq k$;

(iii) $J_{i j} \circ J_{k l}=\{0\}$, if $\{i, j\} \cap\{k, l\}=\varnothing$.

Based on Theorems 2.1 and 2.2, we will introduce a decomposition result for the space $\mathcal{V}$ with respect to a point $z \in \mathcal{V}$. First, we need the following two important operators. For each $z \in \mathcal{V}$, we define the Lyapunov transformation $\mathcal{L}(z): \mathcal{V} \rightarrow \mathcal{V}$ by $\mathcal{L}(z) x=z \circ x$ for all $x \in \mathcal{V}$, which is a symmetric self-adjoint operator in the sense that $\langle\mathcal{L}(z) x, s\rangle=\langle x, \mathcal{L}(z) s\rangle$ for all $x, s \in \mathcal{V}$. The operator $\mathcal{Q}(z):=2 \mathcal{L}^{2}(z)-\mathcal{L}\left(z^{2}\right)$ is called the quadratic representation of $z$. We say two elements $x, s \in \mathcal{V}$ operator commute if $\mathcal{L}(x) \mathcal{L}(s)=\mathcal{L}(s) \mathcal{L}(x)$. By Lemma X.2.2 in [20], two elements $x, s$ operator commute if and only if they share a common Jordan frame. In the matrix algebra of Hermitian matrices, this corresponds to two matrices admitting a simultaneous diagonalization with respect to an orthogonal basis.

In what follows, let $z=\sum_{i=1}^{r} \lambda_{i}(z) q_{i}$ with $\lambda_{1}(z) \geq \lambda_{2}(z) \geq \cdots \geq \lambda_{r}(z)$ and define

$$
\alpha:=\left\{i: \lambda_{i}(z)>0\right\}, \beta:=\left\{i: \lambda_{i}(z)=0\right\} \text { and } \gamma:=\left\{i: \lambda_{i}(z)<0\right\} .
$$

For the Jordan frame $\left\{q_{1}, q_{2}, \cdots, q_{r}\right\}$ and $i, j \in\{1,2, \cdots, r\}$, let $P_{i j}$ be the orthogonal projection operator onto the subspace $J_{i j}$. Then, by Theorem 2.2 , we have

$$
P_{j j}=\mathcal{Q}\left(q_{j}\right) \text { and } P_{i j}=4 \mathcal{L}\left(q_{i}\right) \mathcal{L}\left(q_{j}\right)=4 \mathcal{L}\left(q_{j}\right) \mathcal{L}\left(q_{i}\right)=P_{j i}, i \neq j, i, j \in\{1,2, \cdots, r\},
$$

and the orthogonal projection operators $\left\{P_{i j}: i, j \in\{1,2, \cdots, r\}\right\}$ form a complete system, i.e., they satisfy

$$
P_{i j}=P_{i j}^{*}, P_{i j}^{2}=P_{i j}, P_{i j} P_{k l}=0 \text { if }\{i, j\} \neq\{k, l\}, \sum_{1 \leq i \leq j \leq r} P_{i j}=\mathcal{I},
$$

where $P_{i j}^{*}$ is the adjoint operator of $P_{i j}$ and $\mathcal{I}$ is the identity operator. For a more detailed exposition, see $[19,25]$. By (10), defining six projection operators as

$$
\begin{aligned}
P_{\alpha \alpha} & :=\sum_{i \leq j, i \in \alpha, j \in \alpha} P_{i j}, \quad P_{\alpha \beta}:=\sum_{i \leq j, i \in \alpha, j \in \beta} P_{i j}, \quad P_{\alpha \gamma}:=\sum_{i \leq j, i \in \alpha, j \in \gamma} P_{i j}, \\
P_{\beta \beta} & :=\sum_{i \leq j, i \in \beta, j \in \beta} P_{i j}, \quad P_{\beta \gamma}:=\sum_{i \leq j, i \in \beta, j \in \gamma} P_{i j}, \quad P_{\gamma \gamma}:=\sum_{i \leq j, i \in \gamma, j \in \gamma} P_{i j},
\end{aligned}
$$

we obtain that the above six projection operators are orthogonal and form a complete system, too. Likewise, we specify the following six subspaces as

$$
\begin{aligned}
& J_{\alpha \alpha}:=\bigoplus_{i \leq j, i \in \alpha, j \in \alpha} J_{i j}, \quad J_{\alpha \beta}:=\bigoplus_{i \leq j, i \in \alpha, j \in \beta} J_{i j}, \quad J_{\alpha \gamma}:=\bigoplus_{i \leq j, i \in \alpha, j \in \gamma} J_{i j}, \\
& J_{\beta \beta}:=\bigoplus_{i \leq j, i \in \beta, j \in \beta} J_{i j}, \quad J_{\beta \gamma}:=\bigoplus_{i \leq j, i \in \beta, j \in \gamma} J_{i j}, \quad J_{\gamma \gamma}:=\bigoplus_{i \leq j, i \in \gamma, j \in \gamma} J_{i j} .
\end{aligned}
$$


Since $J_{i j}=P_{i j} \mathcal{V}$, the above subspaces are well-defined and it is easy to see that

$$
J_{\alpha \alpha}=P_{\alpha \alpha} \mathcal{V}, \quad J_{\alpha \beta}=P_{\alpha \beta} \mathcal{V}, \quad J_{\alpha \gamma}=P_{\alpha \gamma} \mathcal{V}, \quad J_{\beta \beta}=P_{\beta \beta} \mathcal{V}, \quad J_{\beta \gamma}=P_{\beta \gamma} \mathcal{V}, \quad J_{\gamma \gamma}=P_{\gamma \gamma} \mathcal{V}
$$

Summarizing the above construction, we have the following decomposition result, which is useful in the subsequent analysis.

Theorem 2.3 Let $\mathcal{V}=(\mathcal{J},\langle\cdot, \cdot\rangle, \circ)$ be a Euclidean Jordan algebra with rank $r$. For $z \in \mathcal{V}$, let $z=\sum_{i=1}^{r} \lambda_{i}(z) q_{i}$ with $\lambda_{1}(z) \geq \lambda_{2}(z) \geq \cdots \geq \lambda_{r}(z)$. Suppose that the index sets $\alpha, \beta, \gamma$ are given by (8). Then $\mathcal{V}$ is the orthogonal direct sum of six subspaces

$$
\mathcal{V}=J_{\alpha \alpha} \oplus J_{\alpha \beta} \oplus J_{\alpha \gamma} \oplus J_{\beta \beta} \oplus J_{\beta \gamma} \oplus J_{\gamma \gamma},
$$

where $J_{\alpha \alpha}, J_{\alpha \beta}, J_{\alpha \gamma}, J_{\beta \beta}, J_{\beta \gamma}, J_{\gamma \gamma}$ are specified by (12). Moreover, these six subspaces are uniquely determined by the three elements: $\sum_{i \in \alpha} q_{i}, \sum_{i \in \beta} q_{i}$ and $\sum_{i \in \gamma} q_{i}$, which are invariant under different choices of the Jordan frames in $\mathcal{C}(z)$.

Furthermore, $\left(J_{\alpha \alpha},\langle\cdot, \cdot\rangle, \circ\right),\left(J_{\beta \beta},\langle\cdot, \cdot\rangle, \circ\right)$ and $\left(J_{\gamma \gamma},\langle\cdot, \cdot\rangle, \circ\right)$ form Euclidean Jordan algebras with rank $|\alpha|,|\beta|$ and $|\gamma|$ and identity elements $\sum_{i \in \alpha} q_{i}, \sum_{i \in \beta} q_{i}$ and $\sum_{i \in \gamma} q_{i}$, respectively.

Proof. By the assumptions and (9) and (12), direct calculation yields

$$
\begin{aligned}
P_{\alpha \alpha} & =\sum_{i<j, i \in \alpha, j \in \alpha} P_{i j}+\sum_{i \in \alpha} P_{i i} \\
& =\sum_{i<j, i \in \alpha, j \in \alpha} 4 \mathcal{L}\left(q_{i}\right) \mathcal{L}\left(q_{j}\right)+\sum_{i \in \alpha}\left(2 \mathcal{L}\left(q_{i}\right) \mathcal{L}\left(q_{i}\right)-\mathcal{L}\left(q_{i}\right)\right) \\
& =\sum_{i \neq j, i \in \alpha, j \in \alpha} 2 \mathcal{L}\left(q_{i}\right) \mathcal{L}\left(q_{j}\right)+\sum_{i \in \alpha} 2 \mathcal{L}\left(q_{i}\right) \mathcal{L}\left(q_{i}\right)-\sum_{i \in \alpha} \mathcal{L}\left(q_{i}\right) \\
& =\sum_{i \in \alpha, j \in \alpha} 2 \mathcal{L}\left(q_{i}\right) \mathcal{L}\left(q_{j}\right)-\mathcal{L}\left(\sum_{i \in \alpha} q_{i}\right) \\
& =2 \mathcal{L}\left(\sum_{i \in \alpha} q_{i}\right) \mathcal{L}\left(\sum_{j \in \alpha} q_{j}\right)-\mathcal{L}\left(\left(\sum_{i \in \alpha} q_{i}\right)^{2}\right) \\
& =\mathcal{Q}\left(\sum_{i \in \alpha} q_{i}\right) .
\end{aligned}
$$

In the same way, we have

$$
P_{\beta \beta}=\mathcal{Q}\left(\sum_{i \in \beta} q_{i}\right), \quad P_{\gamma \gamma}=\mathcal{Q}\left(\sum_{i \in \gamma} q_{i}\right) .
$$

On the other hand, it is easy to see that

$$
\begin{aligned}
P_{\alpha \beta} & =\sum_{i \in \alpha, j \in \beta} 4 \mathcal{L}\left(q_{i}\right) \mathcal{L}\left(q_{j}\right) \\
& =4 \mathcal{L}\left(\sum_{i \in \alpha} q_{i}\right) \mathcal{L}\left(\sum_{j \in \beta} q_{j}\right) .
\end{aligned}
$$


Similarly, we have

$$
P_{\alpha \gamma}=4 \mathcal{L}\left(\sum_{i \in \alpha} q_{i}\right) \mathcal{L}\left(\sum_{j \in \gamma} q_{j}\right), \quad P_{\beta \gamma}=4 \mathcal{L}\left(\sum_{i \in \beta} q_{i}\right) \mathcal{L}\left(\sum_{j \in \gamma} q_{j}\right) .
$$

From the argument after Theorem 2.1, we obtain that $\sum_{i \in \alpha} q_{i}, \sum_{i \in \beta} q_{i}$ and $\sum_{i \in \gamma} q_{i}$ are uniquely determined by $z$, which are invariant under different choices of the Jordan frames in $\mathcal{C}(z)$. Thus, the first part of the theorem follows from Theorem 2.2 and (12) and (13). Note that, by (14) and (15) or by Lemma 20 in [2], it follows that

$$
J_{\alpha \alpha}=J\left(\sum_{i \in \alpha} q_{i}, 1\right), J_{\beta \beta}=J\left(\sum_{i \in \beta} q_{i}, 1\right), J_{\gamma \gamma}=J\left(\sum_{i \in \gamma} q_{i}, 1\right) .
$$

Then, by Theorem 2.2 and (18), we can verify the second part by direct calculation.

As a direct consequence of the above theorem, we easily have the following.

Proposition 2.4 Every $w \in \mathcal{V}$ can be expressed as

$$
w=w_{\alpha \alpha}+w_{\alpha \beta}+w_{\alpha \gamma}+w_{\beta \beta}+w_{\beta \gamma}+w_{\gamma \gamma}
$$

where $w_{\alpha \alpha} \in J_{\alpha \alpha}, w_{\alpha \beta} \in J_{\alpha \beta}, w_{\alpha \gamma} \in J_{\alpha \gamma}, w_{\beta \beta} \in J_{\beta \beta}, w_{\beta \gamma} \in J_{\beta \gamma}, w_{\gamma \gamma} \in J_{\gamma \gamma}$.

As in the proof of Theorem 2.3, we can obtain that triples $\left(J_{\alpha \alpha} \bigoplus J_{\alpha \beta} \bigoplus J_{\beta \beta},\langle\cdot, \cdot\rangle, \circ\right)$, $\left(J_{\beta \beta} \bigoplus J_{\beta \gamma} \bigoplus J_{\gamma \gamma},\langle\cdot, \cdot\rangle, \circ\right)$ and $\left(J_{\alpha \alpha} \bigoplus J_{\alpha \gamma} \bigoplus J_{\gamma \gamma},\langle\cdot, \cdot\rangle, \circ\right)$ form Euclidean Jordan algebras with rank $|\alpha \cup \beta|,|\beta \cup \gamma|$ and $|\alpha \cup \gamma|$ and identity elements $\sum_{i \in \alpha \cup \beta} q_{i}, \sum_{i \in \beta \cup \gamma} q_{i}$ and $\sum_{i \in \alpha \cup \gamma} q_{i}$, respectively. In what follows, let $K^{|\alpha|}, K^{|\beta|}, K^{|\gamma|}, K^{|\alpha \cup \beta|}, K^{|\beta \cup \gamma|}$ and $K^{|\alpha \cup \gamma|}$ respectively be the corresponding symmetric cones in Euclidean Jordan algebras $\left(J_{\alpha \alpha},\langle\cdot, \cdot\rangle, \circ\right),\left(J_{\beta \beta},\langle\cdot, \cdot\rangle, \circ\right),\left(J_{\gamma \gamma},\langle\cdot, \cdot\rangle, \circ\right)$, $\left(J_{\alpha \alpha} \oplus J_{\alpha \beta} \bigoplus J_{\beta \beta},\langle\cdot, \cdot\rangle, \circ\right),\left(J_{\beta \beta} \bigoplus J_{\beta \gamma} \bigoplus J_{\gamma \gamma},\langle\cdot, \cdot\rangle, \circ\right)$ and $\left(J_{\alpha \alpha} \bigoplus J_{\alpha \gamma} \oplus J_{\gamma \gamma},\langle\cdot, \cdot\rangle, \circ\right)$.

\subsection{Generalized Jacobian}

In this subsection, we give a triangular representation of the Jacobian of Löwner operator, and Clarke's generalized Jacobian of metric projection operator $\Pi_{K}$. The latter plays a role in establishing some basic concepts such as the tangent cone and lineality space of $K$ at $z_{+}$. Here, we assume that the reader is familiar with the concepts of (strong) semismoothness, and refer to $[21,22,23]$ for details.

Suppose that the scalar function $g$ is differentiable on $\mathbb{R}$. Let $\lambda:=\left(\lambda_{1}, \lambda_{2}, \cdots, \lambda_{r}\right)^{T} \in \mathbb{R}^{r}$. Recall that the first divided difference $g^{[1]}(\lambda)$ of $g$ at $\lambda$ is the $r \times r$ symmetric matrix, where the $i j$-th entry is given by

$$
\left[\lambda_{i}, \lambda_{j}\right]_{g}:=\left\{\begin{array}{cc}
\frac{g\left(\lambda_{i}\right)-g\left(\lambda_{j}\right)}{\lambda_{i}-\lambda_{j}} & \text { if } \lambda_{i} \neq \lambda_{j} \\
g^{\prime}\left(\lambda_{i}\right) & \text { if } \lambda_{i}=\lambda_{j}
\end{array}\right.
$$

We define the corresponding triangle part $\operatorname{Tri}\left(g^{[1]}(\lambda)\right)$ of $g^{[1]}(\lambda)$ as

$$
\left(\operatorname{Tri}\left(g^{[1]}(\lambda)\right)\right)_{i j}:=\left\{\begin{array}{cl}
\left(g^{[1]}(\lambda)\right)_{i j} & \text { if } i \leq j \\
0 & \text { if } i>j
\end{array}\right.
$$


By (11), define a triangular operator as $P:=\left(P_{i j}\right)$ with $i \leq j$ where $P_{i j}$ is the projection operator onto the subspace $J_{i j}$. Then, we may define a generalized, weighted partial trace $\odot$ of an ordered pair of operators of $\operatorname{Tri}\left(g^{[1]}(\lambda)\right)$ and $P$ as a new operator:

$$
\operatorname{Tri}\left(g^{[1]}(\lambda)\right) \odot P \quad:=\sum_{1 \leq i \leq j \leq r}\left[\tau_{i}, \tau_{j}\right]_{g} P_{i j}
$$

This is a generalization of the partial trace operator in the sense that if the operator $\operatorname{Tri}\left(g^{[1]}(\lambda)\right)$ is representable by a 0,1 matrix then $\operatorname{Tri}\left(g^{[1]}(\lambda)\right) \odot P$ becomes a partial trace of $P$. For the notion of partial trace, see, e.g., [24].

Based on Theorem 3.2 of [25] and Theorem 2.8 of [26], we directly obtain the following Jacobian properties of the Löwner operator $G(\cdot)$.

Theorem 2.5 Let $z=\sum_{j=1}^{r} \lambda_{j}(z) q_{j}$. Then, $G(\cdot)$ is (continuously) differentiable at $z$ if and only if for each $j \in\{1,2, \cdots, r\}, g$ is (continuously) differentiable at $\lambda_{j}(z)$. In this case, the Jacobian $\nabla G(z)$ is given by

$$
\nabla G(z)=\operatorname{Tri}\left(g^{[1]}(\lambda(z))\right) \odot P=\sum_{1 \leq i \leq j \leq r}\left[\lambda_{i}(z), \lambda_{j}(z)\right]_{g} P_{i j} .
$$

We call (22) the triangular representation of the Jacobian $\nabla G(z)$.

Recently, Sun and Sun [25] showed that the metric projection $\Pi_{K}$ onto symmetric cones is strongly semismooth everywhere. Employing a matrix representation approach, Kong, Tunçel and Xiu [27] presented an exact expression for Clarke's generalized Jacobian of $\Pi_{K}$, which is linked to rank-one matrices. Based on the work mentioned above and Theorem 2.3, we will formulate a triangular representation for Clarke's generalized Jacobian of $\Pi_{K}$.

Let $z:=\sum_{i=1}^{r} \lambda_{i}(z) q_{i}$ and the index sets $\alpha, \beta, \gamma$ be given by (8). We recall a set of matrices $\Lambda_{t}(z)$ from [27]. For a given integer $t \in\{0,1, \cdots,|\beta|\}$, we define a set of $r \times r$ matrices $\Lambda_{t}(z)$ by

$$
\Lambda_{t}(z):=\left\{\left(\begin{array}{ccc}
E_{|\alpha| \times|\alpha|} & E_{|\alpha| \times|\beta|} & \Gamma_{|\alpha| \times|\gamma|} \\
E_{|\alpha| \times|\beta|}^{T} & \Lambda_{0} & 0_{|\beta| \times|\gamma|} \\
\Gamma_{|\alpha| \times|\gamma|}^{T} & 0_{|\gamma| \times|\beta|} & 0_{|\gamma| \times|\gamma|}
\end{array}\right): \Lambda_{0}=\left(\begin{array}{cc}
E & \Lambda_{00} \\
\Lambda_{00}^{T} & 0
\end{array}\right), \Lambda_{00} \in \Lambda(t,|\beta|)\right\},
$$

where $E_{|\alpha| \times|\alpha|}$ and $E_{|\alpha| \times|\beta|}$ are the all ones matrix, $\Gamma_{|\alpha| \times|\gamma|}$ is a given matrix by the $i j$-entry $\Gamma_{i j}=\frac{\lambda_{i}(z)}{\lambda_{i}(z)-\lambda_{j}(z)}(i \in \alpha, j \in \gamma)$, and $\Lambda(t,|\beta|)$ is a set of $t \times(|\beta|-t)$ matrices $\left(\theta_{i j}\right)_{t \times(|\beta|-t)}$ (the rows are indexed by $|\alpha|+1,|\alpha|+2, \cdots,|\alpha|+t$, and the columns are indexed by $|\alpha|+t+1,|\alpha|+$ $t+2, \cdots,|\alpha|+|\beta|)$ specified by

$$
\Lambda(t,|\beta|):=\left\{\left(\theta_{i j}\right)_{t \times(|\beta|-t)} \in[0,1]^{t \times(|\beta|-t)}: \theta_{i j} \text { satisfy }(a) \text { and }(b) \text { below }\right\}
$$

(a) $\theta_{i,|\alpha|+t+1} \geq \theta_{i,|\alpha|+t+2} \geq \cdots \geq \theta_{i,|\alpha|+|\beta|}(i \in\{|\alpha|+1,|\alpha|+2, \cdots,|\alpha|+t\})$,

$$
\theta_{|\alpha|+1, j} \geq \theta_{|\alpha|+2, j} \geq \cdots \geq \theta_{|\alpha|+t, j}(j \in\{|\alpha|+t+1,|\alpha|+t+2, \cdots,|\alpha|+|\beta|\}) ;
$$

(b) $\left(\frac{1}{\theta_{i j}}-1\right)_{t \times(|\beta|-t)}$ is a matrix of rank at most one.

Based on the above set of matrices, we define a set of (upper) triangular matrices $U_{t}(z)$ as

$$
U_{t}(z):=\left\{U: U_{i j}=0(i>j), U+U^{T}-\operatorname{Diag}\{\underbrace{1, \cdots, 1}_{|\alpha|+t}, 0, \cdots, 0\} \in \Lambda_{t}(z)\right\} .
$$


As in (11) and (12), we block partition the triangular matrix $U \in U_{t}(z)$ into three triangular submatrices $U_{\alpha \alpha}, U_{\beta \beta}, U_{\gamma \gamma}$ and three other submatrices $U_{\alpha \beta}, U_{\alpha \gamma}, U_{\beta \gamma}$. That is,

$$
U:=\left(\begin{array}{ccc}
U_{\alpha \alpha} & U_{\alpha \beta} & U_{\alpha \gamma} \\
& U_{\beta \beta} & U_{\beta \gamma} \\
& & U_{\gamma \gamma}
\end{array}\right) .
$$

We rewrite the corresponding triangular operator as

$$
P:=\left(\begin{array}{ccc}
P_{\alpha \alpha} & P_{\alpha \beta} & P_{\alpha \gamma} \\
& P_{\beta \beta} & P_{\beta \gamma} \\
& & P_{\gamma \gamma}
\end{array}\right) .
$$

Similarly, we define the following generalized, weighted partial trace $\odot$ of ordered pairs of operators as:

$$
\begin{aligned}
U_{\alpha \alpha} \odot P_{\alpha \alpha} & :=\sum_{i \leq j, i, j \in \alpha} U_{i j} P_{i j}, \quad U_{\alpha \beta} \odot P_{\alpha \beta}:=\sum_{i \in \alpha, j \in \beta} U_{i j} P_{i j}, \quad U_{\alpha \gamma} \odot P_{\alpha \gamma}:=\sum_{i \in \alpha, j \in \gamma} U_{i j} P_{i j}, \\
U_{\beta \beta} \odot P_{\beta \beta} & :=\sum_{i \leq j, i, j \in \beta} U_{i j} P_{i j}, \quad U_{\beta \gamma} \odot P_{\beta \gamma}:=\sum_{i \in \beta, j \in \gamma} U_{i j} P_{i j}, \quad U_{\gamma \gamma} \odot P_{\gamma \gamma}:=\sum_{i \leq j, i, j \in \gamma} U_{i j} P_{i j}, \\
U \odot P & :=U_{\alpha \alpha} \odot P_{\alpha \alpha}+U_{\alpha \beta} \odot P_{\alpha \beta}+U_{\alpha \gamma} \odot P_{\alpha \gamma}+U_{\beta \beta} \odot P_{\beta \beta}+U_{\beta \gamma} \odot P_{\beta \gamma}+U_{\gamma \gamma} \odot P_{\gamma \gamma} \\
& =\sum_{1 \leq i \leq j \leq r} U_{i j} P_{i j} .
\end{aligned}
$$

As a natural continuation of Proposition 2.5 and Theorem 3.1 of [27], we are ready to give the formula for Clarke's generalized Jacobian of $\Pi_{K}$ at $z$, which states the interesting connection between $\partial_{B} \Pi_{K}(z)$ (respectively, $\partial \Pi_{K}(z)$ ) and $\partial_{B} \Pi_{K^{|\beta|}}(0)$ (respectively, $\partial \Pi_{K^{|\beta|}}(0)$ ). In the case of $\mathbb{S}^{n}$, it reduces to Proposition 2.2 of [12].

Proposition 2.6 Let $z=\sum_{i=1}^{r} \lambda_{i}(z) q_{i}$ and the index sets $\alpha, \beta, \gamma$ be given by (8). The $B$ subdifferential of $\Pi_{K}(\cdot)$ at $z$ is given by

$$
\partial_{B} \Pi_{K}(z)=P_{\alpha \alpha}+P_{\alpha \beta}+U_{\alpha \gamma} \odot P_{\alpha \gamma}+\partial_{B} \Pi_{K|\beta|}(0) .
$$

Furthermore, Clarke's generalized Jacobian of $\Pi_{K}(\cdot)$ at $z$ is

$$
\partial \Pi_{K}(z)=\operatorname{conv}\left\{\partial_{B} \Pi_{K}(z)\right\}=P_{\alpha \alpha}+P_{\alpha \beta}+U_{\alpha \gamma} \odot P_{\alpha \gamma}+\partial \Pi_{K|\beta|}(0) .
$$

It is known from Proposition 3.3 of [25] that $\Pi_{K}$ is strongly semismooth on $\mathcal{V}$. For any $d \in \mathcal{V}$, let $d_{i j}:=P_{i j} d$, and

$$
U_{\alpha \gamma} \odot d_{\alpha \gamma}:=\left[U_{\alpha \gamma} \odot P_{\alpha \gamma}\right] d=\sum_{i \in \alpha, j \in \gamma} U_{i j} d_{i j} .
$$

Noting that

$$
\Pi_{K|\beta|}^{\prime}\left(d_{\beta \beta} ; d\right)=\lim _{t \downarrow 0} \frac{\Pi_{K^{|\beta|}}\left(d_{\beta \beta}+t d\right)-\Pi_{K|\beta|}\left(d_{\beta \beta}\right)}{t}=\Pi_{K|\beta|}\left(d_{\beta \beta}\right),
$$

we will give the directional derivative of $\Pi_{K}$ at $z$ along the direction $d$, which reduces to a well-known result of Sun and Sun [28] in the setting of $\mathbb{S}^{n}$. 
Proposition 2.7 Let $z=\sum_{i=1}^{r} \lambda_{i}(z) q_{i}$ and the index sets $\alpha, \beta, \gamma$ be given by (8). Then the directional derivative of $\Pi_{K}$ at $z$ along the direction $d$ is specified by

$$
\Pi_{K}^{\prime}(z ; d)=d_{\alpha \alpha}+d_{\alpha \beta}+U_{\alpha \gamma} \odot d_{\alpha \gamma}+\Pi_{K|\beta|}\left(d_{\beta \beta}\right),
$$

where $U_{\alpha \gamma}$ is given by (24) and (25).

Proof. By assumption and Proposition 2.4, for any given $z \in \mathcal{V}$, let $d=d_{\alpha \alpha}+d_{\alpha \beta}+d_{\alpha \gamma}+$ $d_{\beta \beta}+d_{\beta \gamma}+d_{\gamma \gamma}$. Let $D_{\Pi_{K}|\beta|}$ be the set of the points where $\Pi_{K|\beta|}$ is differentiable. Since $\Pi_{K}$ is strongly semismooth, $\Pi_{K}^{\prime}(z ; \cdot)$ is well-defined on $\mathcal{V}$.

In order to prove (28), we first consider the case of $d_{\beta \beta} \in D_{\Pi_{K}|\beta|}$ with different eigenvalues $\lambda_{i}\left(d_{\beta \beta}\right)$ in subalgebra $J_{\beta \beta}$. From the definition of strong semismoothness, it follows that for every $V \in \partial_{B} \Pi_{K}(z+t d)$ with positive scalar $t \rightarrow 0$,

$$
\Pi_{K}(z+t d)-\Pi_{K}(z)-V(t d)=O\left(\|t d\|^{2}\right) .
$$

Thus, we have

$$
\lim _{t \downarrow 0} \frac{\Pi_{K}(z+t d)-\Pi_{K}(z)}{t}-\lim _{t \downarrow 0} V d=0
$$

or equivalently,

$$
\Pi_{K}^{\prime}(z ; d)=\lim _{t \downarrow 0} V d
$$

On the other hand, let $z+t d=\sum_{i=1}^{r} \lambda_{i}(z+t d) q_{i}(z+t d)$ with $\lambda_{1}(z+t d) \geq \lambda_{2}(z+t d) \geq \cdots \geq$ $\lambda_{r}(z+t d)$. Note that $\lim _{t \downarrow 0} \lambda(z+t d)=\lambda(z)$. As in (9) and (11), we define

$$
P_{j j}^{\prime}=\mathcal{Q}\left(q_{j}(z+t d)\right) \text { and } P_{i j}^{\prime}=4 \mathcal{L}\left(q_{i}(z+t d)\right) \mathcal{L}\left(q_{j}(z+t d)\right), i \neq j, i, j \in\{1,2, \cdots, r\},
$$

and six projection operators as

$$
\begin{aligned}
& P_{\alpha \alpha}^{\prime}:=\sum_{i \leq j, i \in \alpha, j \in \alpha} P_{i j}^{\prime}, \quad P_{\alpha \beta}^{\prime}:=\sum_{i \leq j, i \in \alpha, j \in \beta} P_{i j}^{\prime}, \quad P_{\alpha \gamma}^{\prime}:=\sum_{i \leq j, i \in \alpha, j \in \gamma} P_{i j}^{\prime}, \\
& P_{\beta \beta}^{\prime}:=\sum_{i \leq j, i \in \beta, j \in \beta} P_{i j}^{\prime}, \quad P_{\beta \gamma}^{\prime}:=\sum_{i \leq j, i \in \beta, j \in \gamma} P_{i j}^{\prime}, \quad P_{\gamma \gamma}^{\prime}:=\sum_{i \leq j, i \in \gamma, j \in \gamma} P_{i j}^{\prime} .
\end{aligned}
$$

By Theorem 2.5 and the argument after it, we have

$$
V=U_{\alpha \alpha}^{\prime} \odot P_{\alpha \alpha}^{\prime}+U_{\alpha \beta}^{\prime} \odot P_{\alpha \beta}^{\prime}+U_{\alpha \gamma}^{\prime} \odot P_{\alpha \gamma}^{\prime}+U_{\beta \beta}^{\prime} \odot P_{\beta \beta}^{\prime}+U_{\beta \gamma}^{\prime} \odot P_{\beta \gamma}^{\prime}+U_{\gamma \gamma}^{\prime} \odot P_{\gamma \gamma}^{\prime},
$$

where $U_{\alpha \alpha}^{\prime}, U_{\alpha \beta}^{\prime}, U_{\alpha \gamma}^{\prime}, U_{\beta \beta}^{\prime}, U_{\beta \gamma}^{\prime}, U_{\gamma \gamma}^{\prime}$ are the submatrices with $U_{i j}^{\prime}=\left[\lambda_{i}(z+t d), \lambda_{j}(z+t d)\right]_{g}$ where $g=(\cdot)_{+} \cdot$ Then

$$
V d=U_{\alpha \alpha}^{\prime} \odot P_{\alpha \alpha}^{\prime} d+U_{\alpha \beta}^{\prime} \odot P_{\alpha \beta}^{\prime} d+U_{\alpha \gamma}^{\prime} \odot P_{\alpha \gamma}^{\prime} d+U_{\beta \beta}^{\prime} \odot P_{\beta \beta}^{\prime} d+U_{\beta \gamma}^{\prime} \odot P_{\beta \gamma}^{\prime} d+U_{\gamma \gamma}^{\prime} \odot P_{\gamma \gamma}^{\prime} d,
$$

and $U_{\beta \beta}^{\prime} \odot P_{\beta \beta}^{\prime} d=\sum_{i \leq j, i \in \beta, j \in \beta}\left[\lambda_{i}(z+t d), \lambda_{j}(z+t d)\right]_{g} P_{i j}^{\prime}$. By Proposition 3.2 in [25], $\lambda(\cdot)$ is strongly semismooth and $\lambda_{i}(z+t d)=\lambda_{i}(z)+\lambda_{i}^{\prime}(z ; t d)+O\left(t^{2}\right)$. By Theorem 4.4.8 of [29], $\lambda_{i}^{\prime}(z ; t d)=\lambda_{i}\left(t d_{\beta \beta}\right)$ for $i \in \beta$. Then we obtain $\lambda_{i}(z+t d)=\lambda_{i}(z)+\lambda_{i}\left(t d_{\beta \beta}\right)+O\left(t^{2}\right)=t \lambda_{i}\left(d_{\beta \beta}\right)+$ $O\left(t^{2}\right)$ for $i \in \beta$. Since $d_{\beta \beta} \in D_{\Pi_{K}|\beta|}$ with different eigenvalues of $d_{\beta \beta}$ in the sub-algebra of $J_{\beta \beta}$, we denote its eigenvalues by $\lambda_{i-|\alpha|}\left(d_{\beta \beta}\right)$ for $i \in \beta$. Then, we easily deduce that for $i, j \in \beta$

$$
\lim _{t \downarrow 0}\left[\lambda_{i}(z+t d), \lambda_{j}(z+t d)\right]_{g}=\lim _{t \downarrow 0} \frac{\left(\lambda_{i}(z+t d)\right)_{+}-\left(\lambda_{j}(z+t d)\right)_{+}}{\lambda_{i}(z+t d)-\lambda_{j}(z+t d)}=\left[\lambda_{i-|\alpha|}\left(d_{\beta \beta}\right), \lambda_{j-|\alpha|}\left(d_{\beta \beta}\right)\right]_{g} .
$$


Therefore, we obtain

$$
\lim _{t \downarrow 0} V d=d_{\alpha \alpha}+d_{\alpha \beta}+U_{\alpha \gamma} \odot d_{\alpha \gamma}+\nabla \Pi_{K^{|\beta|}}\left(d_{\beta \beta}\right) d,
$$

and $\nabla \Pi_{K|\beta|}\left(d_{\beta \beta}\right) d=\Pi_{K^{|\beta|}}^{\prime}\left(d_{\beta \beta} ; d\right)$. This together with $(27)$ prove $(28)$.

In the general case of $d$, applying Lemma 2.2 of [23], we immediately obtain the desired conclusion by the continuity of $\Pi_{K}^{\prime}(z ; \cdot)$.

\subsection{The Tangent Cone}

Employing the previous results and techniques, we now focus on some of the important sets used in describing optimality conditions, such as the tangent cone of $K$ at $z_{+}$. Let $\mathcal{T}_{C}(x)$ and $\mathcal{N}_{C}(x)$ respectively denote the tangent and normal cones to a set $C$ at a point $x \in C$ (see, for instance, Chapter 6 of [30]). If $C$ is convex, they coincide with the corresponding objects in convex analysis $[31]$ and $\mathcal{N}_{C}(x)=-\left[\mathcal{T}_{C}(x)\right]^{*}$.

It is well-known that the tangent cone of the symmetric cone $K$ at $z_{+}, \mathcal{T}_{K}\left(z_{+}\right)$, can be characterized as

$$
\mathcal{T}_{K}\left(z_{+}\right)=\left\{w \in \mathcal{V}: w=\Pi_{K}^{\prime}\left(z_{+} ; w\right)\right\}
$$

where $z=\sum_{i=1}^{r} \lambda_{i}(z) q_{i}$ and the index sets $\alpha, \beta, \gamma$ are given by (8). Observe that $\left(U_{t}\left(z_{+}\right)\right)_{i j}=1$ for $i \in \alpha, j \in \gamma$ and $\lambda_{i}\left(z_{+}\right)=0$ for $i \in \beta \cup \gamma$. From Proposition 2.7, we obtain that

$$
\Pi_{K}^{\prime}\left(z_{+} ; w\right)=w_{\alpha \alpha}+w_{\alpha \beta}+w_{\alpha \gamma}+\Pi_{K|\beta \cup \gamma|}\left(w_{\beta \beta}+w_{\beta \gamma}+w_{\gamma \gamma}\right) .
$$

Therefore, by (32) and (33), we have

$$
\begin{aligned}
\mathcal{T}_{K}\left(z_{+}\right) & =\left\{w \in \mathcal{V}: w_{\beta \beta}+w_{\beta \gamma}+w_{\gamma \gamma} \in K^{|\beta \cup \gamma|}\right\} \\
& =\left\{w \in \mathcal{V}: w_{\beta \beta}+w_{\beta \gamma}+w_{\gamma \gamma} \in K\right\} .
\end{aligned}
$$

Thus,

$$
\mathcal{N}_{K}\left(z_{+}\right)=\left\{w \in \mathcal{V}: w=w_{\beta \beta}+w_{\beta \gamma}+w_{\gamma \gamma} \in(-K)\right\}
$$

Let $\operatorname{lin}\left(\mathcal{T}_{K}\left(z_{+}\right)\right)$denote the lineality space of $\mathcal{T}_{K}\left(z_{+}\right)$, i.e., the largest linear space contained in $\mathcal{T}_{K}\left(z_{+}\right)$. Then, we obtain that

$$
\begin{aligned}
\operatorname{lin}\left(\mathcal{T}_{K}\left(z_{+}\right)\right) & =\left\{w \in \mathcal{V}: w_{\beta \beta}+w_{\beta \gamma}+w_{\gamma \gamma}=0\right\} \\
& =\left\{w \in \mathcal{V}: w_{\beta \beta}=w_{\beta \gamma}=w_{\gamma \gamma}=0\right\}
\end{aligned}
$$

Define the critical cone of $K$ at $z$ as $\mathcal{C}(z ; K):=\mathcal{T}_{K}\left(z_{+}\right) \cap z_{-}^{\perp}$ where $z_{-}^{\perp}:=\left\{w \in \mathcal{V}:\left\langle w, z_{-}\right\rangle=0\right\}$. Clearly, from the fact that $-z_{-} \in J_{\gamma \gamma} \cap K$ by (7), we obtain that

$$
z_{-}^{\perp}=\left\{w \in \mathcal{V}:\left\langle w_{\beta \beta}+w_{\beta \gamma}+w_{\gamma \gamma}, z_{-}\right\rangle=0\right\} .
$$

Therefore, we derive

$$
\begin{aligned}
& \mathcal{C}(z ; K) \\
= & \left\{w \in \mathcal{V}: w_{\beta \beta}+w_{\beta \gamma}+w_{\gamma \gamma} \in K,-z_{-} \in K,\left\langle w_{\beta \beta}+w_{\beta \gamma}+w_{\gamma \gamma},-z_{-}\right\rangle=0\right\} \\
= & \left\{w \in \mathcal{V}: w_{\beta \beta}+w_{\beta \gamma}+w_{\gamma \gamma} \in K,-z_{-} \in K, \quad\left(w_{\beta \beta}+w_{\beta \gamma}+w_{\gamma \gamma}\right) \circ\left(-z_{-}\right)=0\right\} \\
= & \left\{w \in \mathcal{V}: w_{\beta \beta} \in K, w_{\beta \gamma}=0, w_{\gamma \gamma}=0\right\}
\end{aligned}
$$


where the first equality holds by $-z_{-} \in J_{\gamma \gamma} \cap K,(34)$ and (36); the second by Proposition 6 of [2]; the last one is a straightforward calculation using Theorem 2.2. Moreover, letting $\operatorname{aff}(\mathcal{C}(z ; K))$ denote the affine hull of $\mathcal{C}(z ; K)$, we have

$$
\operatorname{aff}(\mathcal{C}(z ; K))=\left\{w \in \mathcal{V}: w_{\beta \gamma}=0, w_{\gamma \gamma}=0\right\}
$$

Summarizing the above arguments, we obtain the following topological result related to $K$.

Proposition 2.8 Let $\mathcal{V}=(\mathcal{J},\langle\cdot, \cdot\rangle, \circ)$ be a Euclidean Jordan algebra with rank $r$. Let $z=$ $\sum_{i=1}^{r} \lambda_{i}(z) q_{i}$ and the index sets $\alpha, \beta, \gamma$ be given by (8). Then, we have the following

$$
\begin{aligned}
\mathcal{T}_{K}\left(z_{+}\right) & =\left\{w \in \mathcal{V}: w_{\beta \beta}+w_{\beta \gamma}+w_{\gamma \gamma} \in K\right\}, \\
\operatorname{lin}\left(\mathcal{T}_{K}\left(z_{+}\right)\right) & =\left\{w \in \mathcal{V}: w_{\beta \beta}=w_{\beta \gamma}=w_{\gamma \gamma}=0\right\}, \\
\mathcal{C}(z ; K) & =\left\{w \in \mathcal{V}: w_{\beta \beta} \in K, w_{\beta \gamma}=0, w_{\gamma \gamma}=0\right\}, \\
\operatorname{aff}(\mathcal{C}(z ; K)) & =\left\{w \in \mathcal{V}: w_{\beta \gamma}=0, w_{\gamma \gamma}=0\right\} .
\end{aligned}
$$

We end this section by presenting the connection between $\mathcal{T}_{K}\left(z_{+}\right)$and the smallest face of $K$ containing $z_{+}$. Recall that we say a convex cone $F \subseteq K$ is a face of $K$ if $w, v \in K, w+v \in F$ imply $w, v \in F$. Let $F$ be a face of $K$, then the complementary face of $F$ is specified by

$$
F^{\Delta}:=\{w \in K:\langle w, x\rangle=0 \quad \forall x \in F\} .
$$

Let $\bar{x}$ be an arbitrary relative interior point of $F$. It is well-known that

$$
F^{\Delta}=\{w \in K:\langle w, \bar{x}\rangle=0\} .
$$

Let face $\left(z_{+}\right)$be the smallest face of $K$ containing $z_{+} \in K$. From the argument following Theorem 2.1 , we obtain

$$
\operatorname{face}\left(z_{+}\right)=\left\{w \in \mathcal{V}: w=w_{\alpha \alpha} \in K\right\}
$$

Thus, we have

$$
\operatorname{face}\left(z_{+}\right)^{\Delta}=\left\{w \in \mathcal{V}: w=w_{\beta \beta}+w_{\beta \gamma}+w_{\gamma \gamma} \in K\right\}
$$

and

$$
\left[\operatorname{face}\left(z_{+}\right)^{\Delta}\right]^{\perp}=\left\{w \in \mathcal{V}: w_{\beta \beta}+w_{\beta \gamma}+w_{\gamma \gamma}=0\right\} .
$$

Therefore, by Proposition 2.8, we actually proved the following.

Proposition 2.9 Let $\mathcal{V}=(\mathcal{J},\langle\cdot, \cdot\rangle, \circ)$ be a Euclidean Jordan algebra with rank $r$. For every $x \in K$, we have

$$
\operatorname{lin}\left(\mathcal{T}_{K}(x)\right)=\left[\operatorname{face}(x)^{\Delta}\right]^{\perp} .
$$

\section{The Main Result}

We will prove our main result which establishes various equivalent conditions for the nonsingularity of Clarke's generalized Jacobian of the KKT system (4) at a KKT point. We begin with the following notations.

Let $\bar{x} \in \mathcal{V}$ be an optimal solution to the SCP problem (1) and define

$$
\mathcal{M}(\bar{x}):=\left\{(y, s) \in \mathbb{R}^{m} \times \mathcal{V}:(\bar{x}, y, s) \text { satisfies the KKT conditions }(3)\right\} .
$$


Let $(\bar{y}, \bar{s}) \in \mathcal{M}(\bar{x})$ and $\bar{z}:=\bar{x}-\bar{s}$. From Proposition 6 of [2] and the fact that $\bar{x} \in K, \bar{s} \in K$ and $\langle\bar{x}, \bar{s}\rangle=0$, we obtain that $\bar{x}=\bar{z}_{+}, \bar{s}=-\bar{z}_{-}=(-\bar{z})_{+}$. Without loss of generality, we assume that $\bar{z}=\sum_{i=1}^{r} \lambda_{i} q_{i}$ with $\lambda_{1} \geq \lambda_{2} \geq \cdots \geq \lambda_{r}$ and define

$$
\bar{\alpha}:=\left\{i: \lambda_{i}>0\right\}, \bar{\beta}:=\left\{i: \lambda_{i}=0\right\} \text { and } \bar{\gamma}:=\left\{i: \lambda_{i}<0\right\} .
$$

For simplicity, let $\alpha, \beta, \gamma$, respectively, replace $\bar{\alpha}, \bar{\beta}, \bar{\gamma}$ (no confusion should arise). In this case, we obtain

$$
\bar{x}=\bar{z}_{+}=\sum_{i \in \alpha} \lambda_{i} q_{i}, \quad \bar{s}=-\bar{z}_{-}=(-\bar{z})_{+}=\sum_{i \in \gamma}\left(-\lambda_{i}\right) q_{i}
$$

Then, by Proposition 2.8, we easily obtain the following:

$$
\begin{aligned}
& \operatorname{lin}\left(\mathcal{T}_{K}(\bar{x})\right)=\operatorname{lin}\left(\mathcal{T}_{K}\left(\bar{z}_{+}\right)\right)=\left\{w \in \mathcal{V}: w_{\beta \beta}=w_{\beta \gamma}=w_{\gamma \gamma}=0\right\}, \\
& \operatorname{lin}\left(\mathcal{T}_{K}(\bar{s})\right)=\operatorname{lin}\left(\mathcal{T}_{K}\left((-\bar{z})_{+}\right)\right)=\left\{w \in \mathcal{V}: w_{\alpha \alpha}=w_{\alpha \beta}=w_{\beta \beta}=0\right\},
\end{aligned}
$$

and

$$
\operatorname{aff}(\mathcal{C}(\bar{z} ; K))=\left\{w \in \mathcal{V}: w_{\beta \gamma}=0, w_{\gamma \gamma}=0\right\} .
$$

Let $\operatorname{Ker}(\mathcal{A})$ denote the Kernel (null space) of the linear operator $\mathcal{A}$. Then, we obtain

$$
\operatorname{aff}(\mathcal{C}(\bar{z} ; K)) \cap \operatorname{Ker}(\mathcal{A})=\left\{w \in \mathcal{V}: \mathcal{A}(w)=0, w_{\beta \gamma}=0, w_{\gamma \gamma}=0\right\} .
$$

Next, we consider an important concept, strong regularity, which was introduced by Robinson [32] for generalized equations. Employing the properties of the normal cone, the KKT system (4) can be rewritten as the following generalized equation:

$$
0 \in\left(\begin{array}{c}
c-\mathcal{A}^{*}(y)-s \\
\mathcal{A}(x)-b \\
x
\end{array}\right)+\left(\begin{array}{c}
\mathcal{N}_{\mathcal{V}}(x) \\
\mathcal{N}_{\mathbb{R}^{m}}(y) \\
\mathcal{N}_{K}(s)
\end{array}\right)
$$

The following strong regularity condition for (44) is adopted from [32].

Definition 3.1 We say that a KKT point $(\bar{x}, \bar{y}, \bar{s}) \in \mathcal{V} \times \mathbb{R}^{m} \times \mathcal{V}$ is a strongly regular solution of the generalized equation (44) if there respectively exist neighborhoods $\mathcal{B}$ and $\aleph$ of the origin 0 in $\mathcal{V} \times \mathbb{R}^{m} \times \mathcal{V}$ and the point $(\bar{x}, \bar{y}, \bar{s})$ such that for every $\zeta \in \mathcal{B}$, the following generalized equation

$$
\zeta \in\left(\begin{array}{c}
c-\mathcal{A}^{*}(y)-s \\
\mathcal{A}(x)-b \\
x
\end{array}\right)+\left(\begin{array}{c}
\mathcal{N}_{\mathcal{V}}(x) \\
\mathcal{N}_{\mathbb{R}^{m}}(y) \\
\mathcal{N}_{K}(s)
\end{array}\right)
$$

has a unique solution in $\aleph$, denoted by $s_{\aleph}(\zeta)$, and the mapping $s_{\aleph}: \mathcal{B} \rightarrow \aleph$ is Lipschitz continuous.

Note that the strong regularity for SDP (or nonlinear SDP) is closely related to another concept called the strong second order sufficient condition, see $[8,12]$ for details and references therein. Motivated by the works mentioned above, we establish the strong second order sufficient condition for the SCP problem (1) based on the following linear-quadratic function.

Definition 3.2 Let $z=\sum_{i=1}^{r} \lambda_{i}(z) q_{i}$ and the index sets $\alpha, \beta, \gamma$ be given by (8). Further let $z^{\dagger}:=\sum_{i \in \alpha \cup \gamma} \frac{1}{\lambda_{i}(z)} q_{i}$. We define the linear-quadratic function $\Upsilon_{z}: \mathcal{V} \times \mathcal{V} \rightarrow \mathbb{R}$ by

$$
\Upsilon_{z}(v, w):=\left\langle v, \mathcal{Q}(w) z^{\dagger}\right\rangle, \quad(v, w) \in \mathcal{V} \times \mathcal{V},
$$

which is linear in the first argument $v$ and quadratic in the second argument $w$. 
Here, we call $z^{\dagger}$ the Moore-Penrose pseudo-inverse of $z$ (and adopt the same notation) since in the context of $\mathbb{S}^{n}$ it is the Moore-Penrose pseudo-inverse of a symmetric matrix. By the definition of operator $\mathcal{Q}$ and the associativity of the inner product, we know that

$$
\begin{aligned}
\Upsilon_{z}(v, w) & =\left\langle v, 2 w \circ\left(w \circ z^{\dagger}\right)-w^{2} \circ z^{\dagger}\right\rangle \\
& =2\left\langle v \circ w, z^{\dagger} \circ w\right\rangle-\left\langle v \circ z^{\dagger}, w^{2}\right\rangle .
\end{aligned}
$$

Clearly, when $v \circ z^{\dagger}=0$, it yields

$$
\Upsilon_{z}(v, w)=2\left\langle v \circ w, z^{\dagger} \circ w\right\rangle=2\left\langle v, w \circ\left(z^{\dagger} \circ w\right)\right\rangle .
$$

For instance, if $(y, s) \in \mathcal{M}(\bar{x})$, then $s \circ \bar{x}^{\dagger}=0$ by the argument in the second paragraph of this section. In what follows, we only apply the above definition in the cases of $(y, s) \in \mathcal{M}(\bar{x})$ and

$$
\Upsilon_{\bar{x}}(s, w)=2\left\langle s \circ w, \bar{x}^{\dagger} \circ w\right\rangle,
$$

which becomes Definition 2.1 of [12] in the context of $\mathbb{S}^{n}$.

Thus, we can give the strong second order sufficient condition for the SCP problem (1).

Definition 3.3 Let $\bar{x}$ be an optimal solution to the SCP problem (1). We say that the strong second order sufficient condition $(S S O S C)$ holds at $\bar{x}$ if

$$
\sup _{(y, s) \in \mathcal{M}(\bar{x})}\left\{\Upsilon_{\bar{x}}(s, w)\right\}>0, \quad \forall 0 \neq w \in \bigcap_{(y, s) \in \mathcal{M}(\bar{x}), z=\bar{x}-s}\{\operatorname{aff}(\mathcal{C}(z ; K)) \cap \operatorname{Ker}(\mathcal{A})\} .
$$

The following result gives a useful and simple characterization of SSOSC when $\mathcal{M}(\bar{x})$ is a singleton, i.e., $\mathcal{M}(\bar{x})=\{(\bar{y}, \bar{s})\}$.

Proposition 3.4 Let $\bar{x}$ be an optimal solution to the SCP problem (1). Assume that $\mathcal{M}(\bar{x})=$ $\{(\bar{y}, \bar{s})\}$. Let $\bar{x}$ and $\bar{s}$ have the spectral decompositions as in (39). Then the SSOSC (45) holds at $\bar{x}$ if and only if the following condition holds

$$
\left[\mathcal{A}(w)=0, w_{\alpha \gamma}=0, w_{\beta \gamma}=0, w_{\gamma \gamma}=0 \Rightarrow w=0,\right] \forall w \in \mathcal{V} .
$$

Proof. Let $\bar{z}:=\bar{x}-\bar{s}:=\sum_{i=1}^{r} \lambda_{i} q_{i}$ with $\lambda_{1} \geq \lambda_{2} \geq \cdots \geq \lambda_{r}$ and the index sets $\alpha, \beta, \gamma$ be given by (8). Note that Proposition 2.4 implies that any $w \in \mathcal{V}$ can be expressed as

$$
w=w_{\alpha \alpha}+w_{\alpha \beta}+w_{\alpha \gamma}+w_{\beta \beta}+w_{\beta \gamma}+w_{\gamma \gamma}
$$

where $w_{\alpha \alpha} \in J_{\alpha \alpha}, w_{\alpha \beta} \in J_{\alpha \beta}, w_{\alpha \gamma} \in J_{\alpha \gamma}, w_{\beta \beta} \in J_{\beta \beta}, w_{\beta \gamma} \in J_{\beta \gamma}, w_{\gamma \gamma} \in J_{\gamma \gamma}$. By the assumptions, we have $\bar{x}=\bar{z}_{+}=\sum_{i \in \alpha} \lambda_{i} q_{i}$ and $\bar{s}=(-\bar{z})_{+}=\sum_{i \in \gamma}\left(-\lambda_{i}\right) q_{i}$. Since $\mathcal{M}(\bar{x})=\{(\bar{y}, \bar{s})\}$, by the argument after Definition 3.2 and (43), it is not hard to see that (45) is equivalent to the following

$$
\left\langle\bar{s} \circ w, \bar{x}^{\dagger} \circ w\right\rangle>0, \quad \forall w \neq 0 \text { such that } \mathcal{A}(w)=0, w_{\beta \gamma}=0, w_{\gamma \gamma}=0 .
$$

Clearly, $\bar{s}=\bar{s}_{\gamma \gamma} \in J_{\gamma \gamma}$ and $\bar{x}^{\dagger}=\sum_{i \in \alpha} \frac{1}{\lambda_{i}} q_{i} \in J_{\alpha \alpha}$. Letting $w_{\beta \gamma}=w_{\gamma \gamma}=0$, by Theorem 2.2, direct calculation yields

$$
\begin{aligned}
\bar{x}^{\dagger} \circ w & =\bar{x}^{\dagger} \circ\left(w_{\alpha \alpha}+w_{\alpha \beta}+w_{\alpha \gamma}+w_{\beta \beta}+w_{\beta \gamma}+w_{\gamma \gamma}\right) \\
& =\bar{x}^{\dagger} \circ\left(w_{\alpha \alpha}+w_{\alpha \beta}\right)+\bar{x}^{\dagger} \circ w_{\alpha \gamma} \\
& =\bar{x}^{\dagger} \circ\left(w_{\alpha \alpha}+w_{\alpha \beta}\right)+\frac{1}{2} \sum_{i \in \alpha, j \in \gamma} \frac{1}{\lambda_{i}} w_{i j},
\end{aligned}
$$


and

$$
\begin{aligned}
\bar{s} \circ w & =\bar{s} \circ\left(w_{\alpha \alpha}+w_{\alpha \beta}+w_{\alpha \gamma}+w_{\beta \beta}+w_{\beta \gamma}+w_{\gamma \gamma}\right) \\
& =\bar{s}_{\gamma \gamma} \circ w_{\alpha \gamma} \\
& =\frac{1}{2} \sum_{i \in \alpha, j \in \gamma}\left(-\lambda_{j}\right) w_{i j} .
\end{aligned}
$$

Therefore, Theorem 2.2 implies that

$$
\begin{aligned}
& \left\langle\bar{x}^{\dagger} \circ w, \bar{s} \circ w\right\rangle \\
= & \left\langle\bar{x}^{\dagger} \circ\left(w_{\alpha \alpha}+w_{\alpha \beta}\right), \frac{1}{2} \sum_{i \in \alpha, j \in \gamma}\left(-\lambda_{j}\right) w_{i j}\right\rangle+\left\langle\frac{1}{2} \sum_{i \in \alpha, j \in \gamma} \frac{1}{\lambda_{i}} w_{i j}, \frac{1}{2} \sum_{i \in \alpha, j \in \gamma}\left(-\lambda_{j}\right) w_{i j}\right\rangle \\
= & \frac{1}{4} \sum_{i \in \alpha, j \in \gamma}\left(\frac{-\lambda_{j}}{\lambda_{i}}\right)\left\|w_{i j}\right\|^{2} .
\end{aligned}
$$

Thus, the SSOSC holds at $\bar{x}$ if and only if the following implication is true

$$
\mathcal{A}(w)=0, w \neq 0, w_{\beta \gamma}=0, w_{\gamma \gamma}=0 \Rightarrow w_{\alpha \gamma} \neq 0, \forall w \in \mathcal{V} .
$$

This is equivalent to the desired conclusion.

From the above proof we also obtain that, for any $w$ with $w_{\beta \gamma}=w_{\gamma \gamma}=0$,

$$
\Upsilon_{\bar{x}}(\bar{s}, w)=\frac{1}{2} \sum_{i \in \alpha, j \in \gamma}\left(\frac{-\lambda_{j}}{\lambda_{i}}\right)\left\|w_{i j}\right\|^{2} .
$$

Continuing the line of thought started with Proposition 3.4, we present a connection between the SSOSC and another important concept of constraint nondegeneracy defined below. Notice that a related concept of nondegeneracy goes back at least to Robinson [33, 34] for a general abstract problem, and the primal and dual nondegeneracy was extensively considered in the SDP context [6, 7, 5, 8, 9, 11, 12], where Bonnans and Shapiro [7] also studied constraint nondegeneracy for general optimization problems. It has been used in $[5,15,35,36]$ for sensitivity

and stability analysis in optimization and variational inequalities. Here, our definition comes from Robinson's terminology [34, 33].

Definition 3.5 We say that the primal constraint nondegeneracy holds at a feasible solution $\bar{x} \in \mathcal{V}$ to the SCP problem (1) if

$$
\left[\begin{array}{l}
\mathcal{A} \\
\mathcal{I}
\end{array}\right] \mathcal{V}+\left[\begin{array}{c}
\{0\} \\
\operatorname{lin}\left(\mathcal{T}_{K}(\bar{x})\right)
\end{array}\right]=\left(\begin{array}{l}
\mathbb{R}^{m} \\
\mathcal{V}
\end{array}\right)
$$

or equivalently

$$
\mathcal{A} \operatorname{lin}\left(\mathcal{T}_{K}(\bar{x})\right)=\mathbb{R}^{m} .
$$

Similarly, we say that the dual constraint nondegeneracy holds at a feasible solution $(\bar{y}, \bar{s}) \in$ $\mathbb{R}^{m} \times \mathcal{V}$ to the dual problem (2) if

$$
\left[\begin{array}{ll}
\mathcal{A}^{*} & \mathcal{I} \\
0 & \mathcal{I}
\end{array}\right]\left(\begin{array}{l}
\mathbb{R}^{m} \\
\mathcal{V}
\end{array}\right)+\left[\begin{array}{c}
\{0\} \\
\operatorname{lin}\left(\mathcal{T}_{K}(\bar{s})\right)
\end{array}\right]=\left(\begin{array}{l}
\mathcal{V} \\
\mathcal{V}
\end{array}\right)
$$

or equivalently

$$
\mathcal{A}^{*} \mathbb{R}^{m}+\operatorname{lin}\left(\mathcal{T}_{K}(\bar{s})\right)=\mathcal{V}
$$


Note that the above definition coincides with the definition of primal and dual nondegeneracy by Pataki [14] in the setting of SDP, also see the reference [5] for details.

Proposition 3.6 Let $\bar{x}$ be an optimal solution to the SCP problem (1). Assume that $\mathcal{M}(\bar{x})=$ $\{(\bar{y}, \bar{s})\}$. Then the SSOSC (45) holds at $\bar{x}$ if and only if the dual constraint nondegeneracy condition (51) holds at $(\bar{y}, \bar{s})$.

Proof. As in the proof of Proposition 3.4, letting $\bar{z}:=\bar{x}-\bar{s}:=\sum_{i=1}^{r} \lambda_{i} q_{i}$ with $\lambda_{1} \geq \lambda_{2} \geq \cdots \geq \lambda_{r}$ and $\alpha, \beta, \gamma$ be defined by (8), we have $\bar{x}=\bar{z}_{+}=\sum_{i \in \alpha} \lambda_{i} q_{i}$ and $\bar{s}=(-\bar{z})_{+}=\sum_{i \in \gamma}\left(-\lambda_{i}\right) q_{i}$. By Proposition 2.4, any $w \in \mathcal{V}$ can be expressed as

$$
w=w_{\alpha \alpha}+w_{\alpha \beta}+w_{\alpha \gamma}+w_{\beta \beta}+w_{\beta \gamma}+w_{\gamma \gamma}
$$

where $w_{\alpha \alpha} \in J_{\alpha \alpha}, w_{\alpha \beta} \in J_{\alpha \beta}, w_{\alpha \gamma} \in J_{\alpha \gamma}, w_{\beta \beta} \in J_{\beta \beta}, w_{\beta \gamma} \in J_{\beta \gamma}, w_{\gamma \gamma} \in J_{\gamma \gamma}$. Note that, since $\mathcal{M}(\bar{x})=\{(\bar{y}, \bar{s})\}$, Proposition 3.4 means that the SSOSC (45) holds at $\bar{x}$ if and only if (46) holds. In order to prove the theorem, we only need to show that (46) holds if and only if the dual constraint nondegeneracy condition (51) holds at $(\bar{y}, \bar{s})$.

First, suppose that (46) holds but the dual constraint nondegeneracy condition (51) does not hold at $(\bar{y}, \bar{s})$. Then,

$$
\left(\mathcal{A}^{*} \mathbb{R}^{m}\right)^{\perp} \cap\left(\operatorname{lin}\left(\mathcal{T}_{K}(\bar{s})\right)\right)^{\perp} \neq\{0\} .
$$

Pick any arbitrary $0 \neq \tilde{w} \in\left(\mathcal{A}^{*} \mathbb{R}^{m}\right)^{\perp} \cap\left(\operatorname{lin}\left(\mathcal{T}_{K}(\bar{s})\right)\right)^{\perp}$. Clearly,

$$
\begin{aligned}
\left\langle\tilde{w}, \mathcal{A}^{*}(y)\right\rangle & =0, \quad \forall y \in \mathbb{R}^{m}, \\
\langle\tilde{w}, s\rangle & =0, \quad \forall s \in \operatorname{lin}\left(\mathcal{T}_{K}(\bar{s})\right) .
\end{aligned}
$$

Note that $\left\langle\tilde{w}, \mathcal{A}^{*}(y)\right\rangle=\langle\mathcal{A}(\tilde{w}), y\rangle$. Then, $(52)$ implies $\mathcal{A}(\tilde{w})=0$. Let

$$
\begin{aligned}
\tilde{w} & =\tilde{w}_{\alpha \alpha}+\tilde{w}_{\alpha \beta}+\tilde{w}_{\alpha \gamma}+\tilde{w}_{\beta \beta}+\tilde{w}_{\beta \gamma}+\tilde{w}_{\gamma \gamma}, \\
s & =s_{\alpha \alpha}+s_{\alpha \beta}+s_{\alpha \gamma}+s_{\beta \beta}+s_{\beta \gamma}+s_{\gamma \gamma} .
\end{aligned}
$$

Thus, by (41) and (53), we have $\tilde{w}_{\alpha \gamma}+\tilde{w}_{\beta \gamma}+\tilde{w}_{\gamma \gamma}=0$, which, together with $(46)$ and $\mathcal{A}(\tilde{w})=0$, yields $\tilde{w}=0$. This is a contradiction. Hence, the dual constraint nondegeneracy condition (51) holds at $(\bar{y}, \bar{s})$.

Conversely, suppose that the dual constraint nondegeneracy condition (51) holds at $(\bar{y}, \bar{s})$. Then, for any $w \in \mathcal{V}$ with $\mathcal{A}(w)=0$ and $w_{\alpha \gamma}+w_{\beta \gamma}+w_{\gamma \gamma}=0$, there exist $y \in \mathbb{R}^{m}$ and $s \in \operatorname{lin}\left(\mathcal{T}_{K}(\bar{s})\right)$ such that $w=\mathcal{A}^{*}(y)+s$. Then, we obtain

$$
\begin{aligned}
\langle w, w\rangle & =\left\langle w, \mathcal{A}^{*}(y)+s\right\rangle \\
& =\langle\mathcal{A}(w), y\rangle+\langle w, s\rangle \\
& =0
\end{aligned}
$$

where the last equality follows from the facts $\mathcal{A}(w)=0$, and $w_{\alpha \gamma}+w_{\beta \gamma}+w_{\gamma \gamma}=0$ and $s_{\alpha \alpha}+$ $s_{\alpha \beta}+s_{\beta \beta}=0$ because $s \in \operatorname{lin}\left(\mathcal{T}_{K}(\bar{s})\right)$. Therefore, $w=0$ as desired. The proof is completed.

Likewise, for the dual SCP problem (2), we can establish a similar statement, which is omitted here for brevity. We remark that in the setting of $\mathbb{S}^{n}$, the above result becomes Proposition 3.8 of $[8]$.

Before giving our main result, we need two lemmas which demonstrate the link between the constraint nondegeneracy and the nonsingularity of Clarke's generalized Jacobian of $H$ at a KKT point $(\bar{x}, \bar{y}, \bar{s}) \in \mathcal{V} \times \mathbb{R}^{m} \times \mathcal{V}$. We first show that the primal and dual constraint nondegeneracy conditions are sufficient for the nonsingularity of any $W \in \partial H(\bar{x}, \bar{y}, \bar{s})$. 
Lemma 3.7 Let $(\bar{x}, \bar{y}, \bar{s}) \in \mathcal{V} \times \mathbb{R}^{m} \times \mathcal{V}$ be a KKT point satisfying the $K K T$ conditions (3) and $H$ be the function given by (4). Suppose that the primal constraint nondegeneracy condition (49) holds at $\bar{x}$ and the dual constraint nondegeneracy condition (51) holds at $(\bar{y}, \bar{s})$. Then, every element in $\partial H(\bar{x}, \bar{y}, \bar{s})$ is nonsingular.

Proof. From Proposition 4.50 of [5] or Theorem 3.5 of [14], we obtain that the primal constraint nondegeneracy condition implies that $\mathcal{M}(\bar{x})=\{(\bar{y}, \bar{s})\}$. Thus, it follows from Proposition 3.6 that the SSOSC holds at $\bar{x}$. That is,

$$
\Upsilon_{\bar{x}}(\bar{s}, w)>0, \quad \forall 0 \neq w \in \operatorname{aff}(\mathcal{C}(\bar{z} ; K)) \cap \operatorname{Ker}(\mathcal{A}),
$$

where $\bar{z}:=\bar{x}-\bar{s}:=\sum_{i=1}^{r} \lambda_{i} q_{i}$ with $\lambda_{1} \geq \lambda_{2} \geq \cdots \geq \lambda_{r}$ and $\alpha, \beta, \gamma$ be defined by (8). Then $\bar{x}=\sum_{i \in \alpha} \lambda_{i} q_{i}, \bar{s}=\sum_{i \in \gamma}\left(-\lambda_{i}\right) q_{i}$. Choose any $W \in \partial H(\bar{x}, \bar{y}, \bar{s})$. We only need to show that $W$ is nonsingular. It is obvious that $W$ has the form

$$
W=\left(\begin{array}{ccc}
0 & -\mathcal{A}^{*} & -\mathcal{I} \\
\mathcal{A} & 0 & 0 \\
\mathcal{I}-V & 0 & V
\end{array}\right)
$$

where $V \in \partial \Pi_{K}(\bar{z})$ is the given element related to $W$. Suppose that $(x, y, s) \in \mathcal{V} \times \mathbb{R}^{m} \times \mathcal{V}$ is any vector such that $W(x, y, s)=0$, i.e.,

$$
\begin{aligned}
-\mathcal{A}^{*}(y)-s & =0 \\
\mathcal{A}(x) & =0 \\
(\mathcal{I}-V) x+V s & =0 .
\end{aligned}
$$

By Proposition 2.4, we can set

$$
\begin{aligned}
& x=x_{\alpha \alpha}+x_{\alpha \beta}+x_{\alpha \gamma}+x_{\beta \beta}+x_{\beta \gamma}+x_{\gamma \gamma}, \\
& s=s_{\alpha \alpha}+s_{\alpha \beta}+s_{\alpha \gamma}+s_{\beta \beta}+s_{\beta \gamma}+s_{\gamma \gamma},
\end{aligned}
$$

where $x_{\alpha \alpha}, s_{\alpha \alpha} \in J_{\alpha \alpha}, x_{\alpha \beta}, s_{\alpha \beta} \in J_{\alpha \beta}, x_{\alpha \gamma}, s_{\alpha \gamma} \in J_{\alpha \gamma}, x_{\beta \beta}, s_{\beta \beta} \in J_{\beta \beta}, x_{\beta \gamma}, s_{\beta \gamma} \in J_{\beta \gamma}, x_{\gamma \gamma}, s_{\gamma \gamma} \in$ $J_{\gamma \gamma}$. By Proposition 2.6, we have

$$
V x=x_{\alpha \alpha}+x_{\alpha \beta}+\sum_{i \in \alpha, j \in \gamma} \frac{\lambda_{i}}{\lambda_{i}-\lambda_{j}} x_{i j}+V_{K|\beta|} x_{\beta \beta},
$$

where $V_{K^{|\beta|}} \in \partial \Pi_{K^{|\beta|}}(0)$ is the element related to $V$. From (57), we obtain that

$$
\begin{aligned}
& x_{\beta \gamma}+x_{\gamma \gamma}=0 \text {, } \\
& s_{\alpha \alpha}+s_{\alpha \beta}=0 \text {, } \\
& \left(\mathcal{I}-V_{K^{|\beta|} \mid}\right) x_{\beta \beta}+V_{K^{|\beta|}} s_{\beta \beta}=0 \text {, } \\
& \sum_{i \in \alpha, j \in \gamma}\left(\frac{-\lambda_{j}}{\lambda_{i}-\lambda_{j}} x_{i j}+\frac{\lambda_{i}}{\lambda_{i}-\lambda_{j}} s_{i j}\right)=0 \text {. }
\end{aligned}
$$

By (61), we have

$$
x_{\beta \beta}=V_{K|\beta|} x_{\beta \beta}-V_{K|\beta|} s_{\beta \beta},
$$

and

$$
0=\left\langle x_{\beta \beta},\left(\mathcal{I}-V_{K^{|\beta|}}\right) x_{\beta \beta}+V_{K^{|\beta|}} s_{\beta \beta}\right\rangle=\left\langle x_{\beta \beta},\left(\mathcal{I}-V_{K^{|\beta|}}\right)_{\beta \beta} x_{\beta \beta}\right\rangle+\left\langle x_{\beta \beta}, V_{K^{|\beta|} \mid} s_{\beta \beta}\right\rangle .
$$


Noting that $\mathcal{I}-V_{K^{|\beta|} \mid}$ is positive semidefinite on the subspace $J_{\beta \beta}$, we obtain

$$
\left\langle x_{\beta \beta}, V_{K|\beta|} s_{\beta \beta}\right\rangle=-\left\langle x_{\beta \beta},\left(\mathcal{I}-V_{K|\beta|}\right) x_{\beta \beta}\right\rangle \leq 0 .
$$

Similarly,

$$
\left\langle V_{K|\beta|} s_{\beta \beta}, s_{\beta \beta}\right\rangle \geq 0
$$

By (63),

$$
\left\langle x_{\beta \beta}, s_{\beta \beta}\right\rangle=\left\langle V_{K|\beta|} x_{\beta \beta}, s_{\beta \beta}\right\rangle-\left\langle V_{K|\beta|} s_{\beta \beta}, s_{\beta \beta}\right\rangle .
$$

This together with (64) and (65) yields that

$$
\left\langle x_{\beta \beta}, s_{\beta \beta}\right\rangle \leq 0 \text {. }
$$

Therefore, we have

$$
\begin{aligned}
\langle x, s\rangle & =\left\langle x_{\alpha \gamma}, s_{\alpha \gamma}\right\rangle+\left\langle x_{\beta \beta}, s_{\beta \beta}\right\rangle \\
& \leq\left\langle x_{\alpha \gamma}, s_{\alpha \gamma}\right\rangle \\
& =\sum_{i \in \alpha, j \in \gamma} \frac{\lambda_{j}}{\lambda_{i}}\left\|x_{i j}\right\|^{2} \\
& =-2 \Upsilon_{\bar{x}}(\bar{s}, x),
\end{aligned}
$$

where the first equality holds by (59) and (60), the second equality follows from $s_{i j}=\frac{\lambda_{j}}{\lambda_{i}} x_{i j}$ for $i \in \alpha, j \in \gamma$ by (62), the third equality holds by (47), and the first inequality holds by (67). This together with (54) and (59) yields $x=0$. Thus, from (55) and (57), we obtain that

$$
s_{\alpha \alpha}+s_{\alpha \beta}+s_{\alpha \gamma}=0, \mathcal{A}^{*}(y)+s=0 .
$$

Note that the constraint nondegeneracy condition (48) means that there exist $v \in \mathcal{V}$ and $w \in$ $\operatorname{lin}\left(\mathcal{T}_{K}(\bar{x})\right)$ such that

$$
\mathcal{A}(v)=y \text { and } v+w=s .
$$

Thus, this together with (54) and $w_{\beta \beta}+w_{\beta \gamma}+w_{\gamma \gamma}=0$ yields

$$
\begin{aligned}
\langle y, y\rangle+\langle s, s\rangle & =\langle y, \mathcal{A}(v)\rangle+\langle s, v+w\rangle \\
& =\left\langle\mathcal{A}^{*}(y), v\right\rangle+\langle s, v\rangle+\langle s, w\rangle \\
& =\left\langle\mathcal{A}^{*}(y)+s, v\right\rangle+\langle s, w\rangle \\
& =0 .
\end{aligned}
$$

Therefore, $s=0$ and $y=0$. Hence, the desired conclusion follows.

Note that $W \in \partial_{B} H(\bar{x}, \bar{y}, \bar{s})$ if and only if there is a $V \in \partial_{B} \Pi_{K}(\bar{z})$ such that

$$
W=\left(\begin{array}{ccc}
0 & -\mathcal{A}^{*} & -\mathcal{I} \\
\mathcal{A} & 0 & 0 \\
\mathcal{I}-V & 0 & V
\end{array}\right)
$$

By Proposition 2.6, 0, $P_{\beta \beta} \in \partial_{B} \Pi_{K^{|\beta|} \mid}(0)$. Let $V^{0}:=P_{\alpha \alpha}+P_{\alpha \beta}+U_{\alpha \gamma} \odot P_{\alpha \gamma}$ and $V^{\mathcal{I}}:=$ $P_{\alpha \alpha}+P_{\alpha \beta}+U_{\alpha \gamma} \odot P_{\alpha \gamma}+P_{\beta \beta}$. Clearly, $V^{0}, V^{\mathcal{I}} \in \partial_{B} \Pi_{K}(\bar{z})$. Let $W^{0}$ and $W^{\mathcal{I}}$ be specified by (69) with $V$ being replaced by $V^{0}$ and $V^{\mathcal{I}}$, respectively. Define $\operatorname{ex}\left(\partial_{B} H(\bar{x}, \bar{y}, \bar{s})\right):=\left\{W^{0}, W^{\mathcal{I}}\right\}$. Clearly, $\operatorname{ex}\left(\partial_{B} H(\bar{x}, \bar{y}, \bar{s})\right) \subseteq \partial_{B} H(\bar{x}, \bar{y}, \bar{s})$.

We continue to show that the nonsingularity of only two elements $W^{0}, W^{\mathcal{I}}$ in $\partial_{B} H(\bar{x}, \bar{y}, \bar{s})$ will imply both the primal and dual constraint nondegeneracy conditions. 
Lemma 3.8 Let $(\bar{x}, \bar{y}, \bar{s}) \in \mathcal{V} \times \mathbb{R}^{m} \times \mathcal{V}$ be a KKT point satisfying the $K K T$ conditions (3) and $H$ be the function given by (4). Suppose that two elements $\left\{W^{0}, W^{\mathcal{I}}\right\}$ of $\operatorname{ex}\left(\partial_{B} H(\bar{x}, \bar{y}, \bar{s})\right)$ are nonsingular. Then the primal constraint nondegeneracy condition (49) holds at $\bar{x}$ and the dual constraint nondegeneracy condition (51) holds at $(\bar{y}, \bar{s})$.

Proof. We consider the following two cases:

Case 1: Assume that the primal constraint nondegeneracy condition (49) does not hold at $\bar{x}$. By Definition 3.5, (48) does not hold. Thus, we obtain

$$
\left\{\left[\begin{array}{l}
\mathcal{A} \\
\mathcal{I}
\end{array}\right] \mathcal{V}\right\}^{\perp} \cap\left[\begin{array}{c}
\{0\} \\
\operatorname{lin}\left(\mathcal{T}_{K}(\bar{x})\right)
\end{array}\right]^{\perp} \neq\{0\} \in\left(\begin{array}{l}
\mathbb{R}^{m} \\
\mathcal{V}
\end{array}\right)
$$

which means that there exists a nonzero $(\hat{y}, \hat{s})$ such that

$$
(\hat{y}, \hat{s}) \in\left\{\left[\begin{array}{c}
\mathcal{A} \\
\mathcal{I}
\end{array}\right] \mathcal{V}\right\}^{\perp}
$$

and

$$
(\hat{y}, \hat{s}) \in\left[\begin{array}{c}
\{0\} \\
\operatorname{lin}\left(\mathcal{T}_{K}(\bar{x})\right)
\end{array}\right]^{\perp}
$$

By $(71)$, for any $w \in \operatorname{lin}\left(\mathcal{T}_{K}(\bar{x})\right)$, we have $\langle\hat{s}, w\rangle=0$. Since $w_{\beta \beta}+w_{\beta \gamma}+w_{\gamma \gamma}=0, \hat{s}_{\alpha \alpha}+\hat{s}_{\alpha \beta}+\hat{s}_{\alpha \gamma}=$ 0 . Then, $V^{0} \hat{s}=\hat{s}_{\alpha \alpha}+\hat{s}_{\alpha \beta}+\left(U_{\alpha \gamma} \odot P_{\alpha \gamma}\right) \hat{s}_{\alpha \gamma}=0$. On the other hand, by (70),

$$
\langle(\hat{y}, \hat{s}),(\mathcal{A}(w), w)\rangle=0, \forall w \in \mathcal{V} \quad \Rightarrow \quad \mathcal{A}^{*}(\hat{y})+\hat{s}=0 .
$$

Letting $x=0$, we obtain that for nonzero $(0, \hat{y}, \hat{s})$

$$
W^{0}(0, \hat{y}, \hat{s})=\left(\begin{array}{ccc}
0 & -\mathcal{A}^{*} & -\mathcal{I} \\
\mathcal{A} & 0 & 0 \\
\mathcal{I}-V^{0} & 0 & V^{0}
\end{array}\right)(0, \hat{y}, \hat{s})=\left(\begin{array}{c}
-\mathcal{A}^{*}(\hat{y})-\hat{s} \\
0 \\
V^{0} \hat{s}
\end{array}\right)=0,
$$

which says that $W^{0}$ is singular. This is a contradiction, and the primal constraint nondegeneracy condition holds.

Case 2: Assume that the dual constraint nondegeneracy condition (51) does not hold at $(\bar{y}, \bar{s})$. We obtain

$$
\left[\mathcal{A}^{*} \mathbb{R}^{m}\right]^{\perp} \cap\left[\operatorname{lin}\left(\mathcal{T}_{K}(\bar{s})\right)\right]^{\perp} \neq\{0\} \in \mathcal{V} .
$$

Similarly, there is a nonzero $\hat{x}$ such that

$$
\hat{x} \in\left[\mathcal{A}^{*} \mathbb{R}^{m}\right]^{\perp},
$$

and

$$
\hat{x} \in\left[\operatorname{lin}\left(\mathcal{T}_{K}(\bar{s})\right)\right]^{\perp} .
$$

By (73) and (41), $\hat{x}_{\alpha \gamma}+\hat{x}_{\beta \gamma}+\hat{x}_{\gamma \gamma}=0$, i.e., $\hat{x}=\hat{x}_{\alpha \alpha}+\hat{x}_{\alpha \beta}+\hat{x}_{\beta \beta}$. Thus, we obtain that $V^{\mathcal{I}} \hat{x}=\hat{x}_{\alpha \alpha}+\hat{x}_{\alpha \beta}+\hat{x}_{\beta \beta}=\hat{x}$, and $\left(\mathcal{I}-V^{\mathcal{I}}\right) \hat{x}=0$. On the other hand, by (72),

$$
\left\langle\hat{x}, \mathcal{A}^{*}(y)\right\rangle=0, \forall y \in \mathbb{R}^{m} \Rightarrow \mathcal{A}(\hat{x})=0 .
$$

Likewise, letting $(y, s)=(0,0)$, we obtain that for nonzero $(\hat{x}, 0,0)$

$$
W^{\mathcal{I}}(\hat{x}, 0,0)=\left(\begin{array}{ccc}
0 & -\mathcal{A}^{*} & -\mathcal{I} \\
\mathcal{A} & 0 & 0 \\
\mathcal{I}-V^{\mathcal{I}} & 0 & V^{\mathcal{I}}
\end{array}\right)(\hat{x}, 0,0)=\left(\begin{array}{c}
0 \\
\mathcal{A}(\hat{x}) \\
\left(\mathcal{I}-V^{\mathcal{I}}\right) \hat{x}
\end{array}\right)=0
$$

which says that $W^{\mathcal{I}}$ is singular. This is also a contradiction, and the dual constraint nondegeneracy condition holds. 
Now, we are in the position to prove our main result.

Theorem 3.9 Let $(\bar{x}, \bar{y}, \bar{s}) \in \mathcal{V} \times \mathbb{R}^{m} \times \mathcal{V}$ be a KKT point satisfying the KKT conditions (3) and $H$ be the function given by (4). Then, the following statements are all equivalent:

(a) Every element in $\partial H(\bar{x}, \bar{y}, \bar{s})$ is nonsingular.

(b) Every element in $\partial_{B} H(\bar{x}, \bar{y}, \bar{s})$ is nonsingular.

(c) Both elements in $\operatorname{ex}\left(\partial_{B} H(\bar{x}, \bar{y}, \bar{s})\right)$ are nonsingular.

(d) The primal constraint nondegeneracy condition (49) holds at $\bar{x}$ and the dual constraint nondegeneracy condition (51) holds at $(\bar{y}, \bar{s})$.

(e) The function $H$ is a locally Lipschitz homeomorphism near $(\bar{x}, \bar{y}, \bar{s})$.

(f) The KKT point $(\bar{x}, \bar{y}, \bar{s})$ is a strongly regular solution of the generalized equation (44).

In each case, $\bar{x}$ is a unique primal optimal solution to $\operatorname{SCP}(1)$ and $(\bar{y}, \bar{s})$ is a unique optimal solution to its dual (2).

Proof. It follows from Lemmas 3.7 and 3.8 that $(b) \Leftrightarrow(a) \Leftrightarrow(c) \Leftrightarrow(d)$. Note that from the proof of Lemma 3.4 of [8], we actually obtain that a similar result is still true in the setting of symmetric cones. This establishes $(e) \Leftrightarrow(f)$. Furthermore, we know from Clarke's inverse function theorem for Lipschitz functions $[21,37]$ that $(b) \Rightarrow(e)$, and from $[38,39]$ that $(e) \Rightarrow(b)$.

Furthermore, by Theorem 3.5 of [14] and the remark after Definition 3.5, if the condition $(d)$ holds then $\bar{x}$ is a unique primal optimal solution and $(\bar{y}, \bar{s})$ is a unique dual optimal solution. Thus, the proof is complete, establishing the equivalence of all statements.

Acknowledgment We thank two anonymous referees for their very useful comments. In particular, one of the referees pointed out a gap in our original proof of Proposition 2.7.

\section{References}

[1] Eaves, B.C.: On the basic theorem of complementarity, Math. Program. 1, pp. 68-75 (1971)

[2] Gowda, M.S., Sznajder, R., Tao, J.: Some P-properties for linear transformations on Euclidean Jordan algebras. Linear Algebra Appl. 393, pp. 203-232 (2004)

[3] Pang, J.-S., Qi, L.: Nonsmooth equations: motivation and algorithms. SIAM J. Optim. 3, pp. 443-465 (1993)

[4] Facchinei, F., Pang, J.-S.: Finite-Dimensional Variational Inequalities and Complementarity Problems. Volume I and II, Springer-Verlag, New York, 2003.

[5] Bonnans, J.F., Shapiro, A.: Perturbation Analysis of Optimization Problems. Springer, New York, 2000.

[6] Alizadeh, F., Haeberly, J.-P. A., Overton, M.L.: Complementarity and nondegeneracy in semidefinite programming. Math. Program. 77, pp. 111-128 (1997)

[7] Bonnans, J.F., Shapiro, A.: Nondegeneracy and quantitative stability of parameterized optimization problems with multiple solutions. SIAM J. Optim. 8, pp. 940-946 (1998)

[8] Chan, Z., Sun, D.: Constraint nondegeneracy, strong regularity and nonsingularity in semidefinite programming. SIAM J. Optim. 19, pp. 370-396 (2008) 
[9] Flegel, M.L., Kanzow, C.: Equivalence of two nondegeneracy conditions for semidefinite programs, J. Optim. Theory Appl. 135, pp. 381-397 (2007)

[10] Kanzow, C., Ferenczi, I., Fukushima, M.: On the local convergence of semismooth Newton methods for linear and nonlinear second-order cone programs without strict complementarity, SIAM J. Optim. 20, pp. 297-320 (2009)

[11] Shapiro, A.: First and second order analysis of nonlinear semidefinite programs. Math. Program. 77, pp. 301-320 (1997)

[12] Sun, D.: The strong second order sufficient condition and constraint nondegeneracy in nonlinear semidefinite programming and their implications. Math. Oper. Res. 31, pp. 761$776(2006)$

[13] Yıldırım, E.A.: An interior-point perspective on sensitivity analysis in semidefinite programming. Math. Oper. Res. 28, pp. 649-676 (2003)

[14] Pataki, G.: The geometry of semidefinite programming. In: Handbook of Semidefinite Programming (H. Wolkowicz, L. Vandenberghe and R. Saigal, ed.), Kluwer, pp. 29-65, 2000

[15] Pataki, G., Tunçel, L.: On the generic properties of convex optimization problems in conic form. Math. Program. A 89, pp. 449-457 (2001)

[16] Yıldırım, E.A.: Unifying optimal partition approach to sensitivity analysis in conic optimization. J. Optim. Theory Appl. 122 (2004), pp. 405-423.

[17] Shapiro, A., Fan, M.K.H.: On eigenvalue optimization, SIAM J. Optim. 5, pp. 552-569 (1995)

[18] Wang, Y.: Perturbation Analysis of Optimization Problems over Symmetric Cones. PhD thesis, Dalian University of Technology, September, 2008.

[19] Koecher, M.: The Minnesota Notes on Jordan Algebras and Their Applications. Edited and annotated by A. Brieg and S. Walcher, Springer, Berlin, 1999.

[20] Faraut, J., Korányi, A.: Analysis on Symmetric Cones. Oxford University Press, New York, 1994.

[21] Clarke, F.H.: Optimization and Nonsmooth Analysis. Wiley, New York, 1983.

[22] Mifflin, R.: Semismooth and semiconvex functions in constrained optimization. SIAM J. Cont. Optim. 15, pp. 957-972 (1977)

[23] Qi, L., Sun, J.: A nonsmooth version of Newton's method. Math. Program. 58, pp. 353-367 (1993)

[24] Nielsen, M.A., Chuang, I.L.: Quantum computation and quantum information. Cambridge, Cambridge University Press, New York, 2000.

[25] Sun, D., Sun, J.: Löwner's operator and spectral functions on Euclidean Jordan algebras. Math. Oper. Res. 33, pp. 421-445 (2008)

[26] Kong, L.C., Sun, J., Xiu, N.H.: A regularized smoothing Newton method for symmetric cone complementarity problems. SIAM J. Optim. 19, pp. 1028-1047 (2008) 
[27] Kong, L.C., Tunçel, L., Xiu, N.H.: Clarke Generalized Jacobian of the Projection onto Symmetric Cones. Set-Valued and Variational Anal. 17, pp. 135-151 (2009)

[28] Sun, D., Sun, J.: Semismooth matrix valued functions. Math. Oper. Res. 27, pp. 150-169 (2002)

[29] Baes, M.: Spectral Functions and Smoothing Techniques on Jordan Algebras. PhD thesis, Université catholique de Louvain, September, 2006.

[30] Rockafellar, R.T., Wets, R.J.-B.: Variational Analysis. Springer, Berlin, 2004.

[31] Rockafellar, R.T.: Convex Analysis. Princeton University Press, Princeton, New Jersey, 1970.

[32] Robinson, S.M.: Strongly regular generalized equations. Math. Oper. Res. 5, pp. 43-62 (1980)

[33] Robinson, S.M.: Local structure of feasible sets in nonlinear programming. Part III: Stability and sensitivity, Mathematical Programming Study, 30, pp. 45-66 (1987)

[34] Robinson, S.M.: Local structure of feasible sets in nonlinear programming. Part II: Nondegeneracy, Mathematical Programming Study, 22, pp. 217-230 (1984)

[35] Robinson, S.M.: Constraint nondegeneracy in variational analysis. Math. Oper. Res. 28, pp. 201-232 (2003)

[36] Shapiro, A.: Sensitivity analysis of generalized equations. J. Math. Sci. 115, pp. 2554-2565 (2003)

[37] Clarke, F.H.: On the inverse function theorem. Pacific J. Math. 64, pp. 97-102 (1976)

[38] Gowda, M.S.: Inverse and implicit function theorems for H-differentiable and semismooth functions. Optim. Methods Softw. 19, pp. 443-461 (2004)

[39] Kummer, B.: Lipschitzian inverse functions, directional derivatives, and applications in $C^{1,1}$ optimization. J. Optim. Theory Appl. 70, pp. 559-580 (1991) 